\title{
Host galaxies of SNe Ic-BL with and without long gamma-ray bursts
}

\author{
J. Japelj ${ }^{1}$, S. D. Vergani ${ }^{2,3}$, R. Salvaterra ${ }^{4}$, M. Renzo ${ }^{1}$, E. Zapartas ${ }^{1}$, S. E. de Mink ${ }^{1}$, L. Kaper ${ }^{1}$, and S. Zibetti ${ }^{5}$ \\ 1 Anton Pannekoek Institute for Astronomy, University of Amsterdam, Science Park 904, 1098 XH Amsterdam, The Netherlands \\ e-mail: j.japelj@uva.nl \\ 2 GEPI - Observatoire de Paris Meudon, 5 Place Jules Jannsen, 92195 Meudon, France \\ 3 INAF - Osservatorio Astronomico di Brera, via E. Bianchi 46, 23807 Merate, Italy \\ ${ }^{4}$ INAF - IASF Milano, via E. Bassini 15, 20133 Milano, Italy \\ 5 INAF - Osservatorio Astrofisico di Arcetri, Largo Enrico Fermi 5, 50125 Firenze, Italy
}

Received 11 April 2018 / Accepted 26 June 2018

\begin{abstract}
Broad-line Ic supernovae (SNe Ic-BL) are a very rare class of core-collapse supernovae exhibiting high ejecta velocities and high kinetic energies. They are the only type of SNe that accompany long gamma-ray burst (GRB) explosions. Systematic differences found in the spectra of SNe Ic-BL with and without GRBs (GRB-SNe and SNe Ic-BL, respectively) could either be due to differences in the progenitor or/and explosion mechanism of SNe Ic-BL caused by the presence or absence of a GRB, or solely to differences in the viewing angle of the observer with respect to the orientation of the collimated explosion. We present the systematic comparison of the host galaxies of broad-lined SNe Ic with and without a detected GRB, the latter being detected in untargeted surveys, with the aim to find out whether there are any systematic differences between the environments in which these two classes of SNe preferentially explode. We study photometric properties of the host galaxies of a sample of $8 \mathrm{GRB}-\mathrm{SNe}$ and a sample of $28 \mathrm{SNe}$ Ic-BL at $z<0.2$. The two galaxy samples have indistinguishable luminosity and proper size distribution. We find indications that GRB-SNe on average occur closer to the centres of their host galaxies, that is, the samples have a different distribution of projected offsets, normalized by the galaxy sizes. In addition, we compare gas-phase metallicities of the GRB-SNe and SNe Ic-BL host samples and find that a larger fraction of super-solar metallicity hosts are found among the SNe Ic-BL without a GRB. Our results are indicative of a genuine difference between the two types of explosions and suggest that the viewing angle is not the main source of difference in the spectra of the two classes. We discuss the implications that our results have on our understanding of progenitors of SNe Ic-BL with and without a GRB.
\end{abstract}

Key words. supernovae: general - gamma-ray burst: general - galaxies: star formation

\section{Introduction}

Massive stars can end their lives in very different ways. Following the collapse of their core and successful ejection of their outer layers (e.g. Woosley et al. 2002), the deaths of massive stars are marked by a variety of core-collapse supernova (SN) explosions (Filippenko 1997). A subclass of core-collapse $\mathrm{SNe}$ are stripped-envelope supernovae, whose progenitor stars have lost most of the hydrogen (Type IIb, $\mathrm{Ib}$ and $\mathrm{Ibn}$ ) or both the hydrogen and helium (Type Ic) in their outer envelopes prior to the collapse. Additionally, some supernovae of Type Ic are found to have very broad lines in their spectra (Type Ic-BL), indicative of very fast ejecta velocities (Modjaz et al. 2016). The detection of Ic-BL SN1998bw following GRB 980425 (Galama et al. 1998) provided a link between the broad-lined supernovae and long gamma-ray burst (GRB) explosions (Kumar \& Zhang 2015), which is now firmly established (Hjorth et al. 2003; Malesani et al. 2004; see e.g. Woosley \& Bloom 2006; Cano et al. 2017b for a review). The subjects of this paper are SNe Ic-BL with and without associated GRBs, referred to in the following as GRB-SNe and $\mathrm{SNe}$ Ic-BL, respectively.

Mass, binarity, metallicity, rotation rate, mass-loss and probably magnetic fields play critical roles in forming evolved objects and determine the final fate of stars (see reviews by e.g. Maeder \& Meynet 2000; Heger et al. 2003; Langer 2012). The fraction of broad-lined SNe Ic compared to the general population of core-collapse $\mathrm{SNe}$ is only $\sim 1 \%$ (e.g. Smith et al. 2011; Graur et al. 2017). Evidently the occurrence of a broadline Ic SN demands special conditions that only a small fraction of massive stars satisfy. The fraction of long GRBs ${ }^{1}$ compared to the core-collapse $\mathrm{SNe}$ is likely even lower (Soderberg et al. 2006; Guetta \& Della Valle 2007; Ghirlanda et al. 2013). However, because the GRB explosions are highly collimated (Granot 2007; Ghirlanda et al. 2013; van Eerten 2018), the GRB rate estimates come with large uncertainties as the typical beaming angles are poorly known.

Due to the collimated GRB explosions it is also not straightforward to understand whether all SNe Ic-BL are linked to a GRB explosion or not: a non-detection of a GRB for a particular SN Ic-BL could simply be due to the explosion being directed away from us. Because these $\mathrm{SNe}$ occur at relatively large distances, it is hard to obtain direct evidence regarding their progenitors: until now only one SN Ib (iPTF13bvn; e.g. Cao et al. 2013; Groh et al. 2013) and potentially one SN Ic (SN2017ein; Van Dyk et al. 2018) progenitor have been detected. Nevertheless, some evidence exists that at least some SNe Ic-BL are not accompanied by a GRB. For example, recent radio studies of large samples (Corsi et al. 2016) show that not all SNe Ic-BL are connected to GRBs (see also Soderberg et al. 2006, 2010). On the other hand, polarimetry (e.g. Wang \& Wheeler 2008)

\footnotetext{
1 In this paper we do not generally distinguish between low- and highluminosity GRBs linked to the GRB-SN (e.g. Soderberg et al. 2006).
} 
and late-time spectroscopy (e.g. Taubenberger et al. 2009) of the $\mathrm{SNe} \mathrm{Ib} / \mathrm{c}$ indicate that all these explosions are to some degree aspherical. The difference between a SN with and without a GRB may therefore be a successful break-out of a jet from the star in the GRB case (Lazzati et al. 2012; Barnes et al. 2018). Supposing that there are two genuine classes of broad-line $\mathrm{SNe}$, that is, those with and without a GRB, the factors leading to the separate outcomes remain to be identified.

More information can be obtained by comparing the SN IcBL and GRB-SN population properties (e.g. Cano et al. 2017b). The number of detected and observed stripped-envelope $\mathrm{SNe}$ (including $\mathrm{SNe}$ Ic-BL) has increased in recent years (e.g. Modjaz et al. 2014, 2016; Prentice et al. 2016; Lyman et al. 2016) mostly thanks to the transient all-sky surveys such as the Palomar Transient Factory (Law et al. 2009). The number of well-studied GRB-SNe is also slowly but steadily rising (e.g. Kann et al. 2016; Cano et al. 2017b), allowing for a statistical comparison between the two populations. Comparing the optical spectra of SNe Ic-BL and GRB-SNe, Modjaz et al. (2016) showed that the two classes present clear differences, with the spectra of GRB-SNe having higher absorption velocities and broader line widths. This difference also shows itself in the higher kinetic energies of the GRB-SNe, as determined from the studies of their bolometric light curves (Cano 2013).

Another line of evidence pointing to the nature of the progenitors lies in their host galaxy environments. For example, the so-called "collapsar" single-star evolution model, in which the progenitors of GRBs are massive stars with a high core rotation rate, indeed predicts that the progenitor star should have low metallicity in order to minimize the angular momentum losses and keep the high rotation rate of the core (e.g. Woosley \& Bloom 2006). However, the gas-phase metallicities found by analysing large samples of GRB host galaxies extend to higher values (e.g. Vergani et al. 2015, 2017; Krühler et al. 2015; Japelj et al. 2016a; Perley et al. 2016b) as predicted by the collapsar model (Yoon et al. 2006). Alternatively, GRBs could be formed in binary progenitor systems (e.g. formation through a common envelope (Tout et al. 2011; Zapartas et al. 2017), tidal interaction (Izzard et al. 2004; Detmers et al. 2008) or from runaway stars (Cantiello et al. 2007; Eldridge et al. 2011)) which would imply the less stringent high-metallicity cut. Furthermore, clues about the progenitors are also found by investigating properties like star formation rate densities (Kelly et al. 2014) or ages of stellar populations in the hosts (e.g. Thöne et al. 2015).

In particular, possible differences in the environments of GRB-SNe and SNe Ic-BL could reveal some properties of their progenitors. Any observed difference in this case cannot be attributed to the effect of the viewing angle. Several studies have focused on the host galaxies of stripped-envelope supernovae (e.g. Modjaz et al. 2008, 2011; Anderson et al. 2010; Sanders et al. 2012; Kelly \& Kirshner 2012; Kelly et al. 2014; Graham \& Fruchter 2013). In these studies, the comparison either included the hosts of SNe detected in targeted surveys (which introduced a bias towards brighter galaxies) or the number of events of each class was low and the SNe Ic-BL with and without GRBs were combined into a single class. In comparison to $\mathrm{SNe} \mathrm{Ib} / \mathrm{c}$, broad-lined supernovae in general were found to prefer environments with lower metallicity. Broadline supernovae are furthermore found in galaxies with higher star-formation rate density with respect to $\mathrm{SNe} \mathrm{Ib} / \mathrm{c}$ (Kelly et al. 2014). The interpretation of the results of the comparison studies is however difficult due to either the small sample size, the inclusion of SNe from targeted surveys in the analysis, or the comparison of populations lying at completely different average redshifts.

In this work, we focus on the comparison of the morphophotometric properties of a sample of untargeted SNe Ic-BL and GRB-SNe host galaxies at $z<0.2$. The data and analysis are presented in Sect. 2. In particular, we compare the galaxy luminosities, sizes, and explosion offsets from the host's centre (Sect. 3). Furthermore, we collect available information of host galaxy spectra from the literature and measure gas-phase metallicities (Sect. 4). In our study, we find evidence that GRB-SNe are found at smaller offsets from galactic centres and in environments of lower metallicities compared to SNe without GRBs. We discuss the implications of these results in Sect. 5. Finally we provide conclusions in Sect. 6.

All errors are reported at $1 \sigma$ confidence unless stated otherwise. We use a standard cosmology (Planck Collaboration XVI 2014): $\Omega_{\mathrm{m}}=0.315, \Omega_{\Lambda}=0.685$, and $H_{0}=67.3 \mathrm{~km} \mathrm{~s}^{-1} \mathrm{Mpc}^{-1}$. All magnitudes are reported in the AB scale.

\section{Data and analysis}

Supernovae can be discovered by targeted or untargeted surveys. Targeted surveys periodically look at a sample of preselected galaxies. This introduces a bias to the host galaxy population towards brighter and more metal-rich host galaxies on average (e.g. Modjaz et al. 2011; Sanders et al. 2012). We therefore include only $\mathrm{SNe}$ discovered by untargeted surveys in our samples. We searched through the available literature and The Open Supernova Catalog $\operatorname{archive}^{2}$ (Guillochon et al. 2017) for all spectroscopically confirmed SNe Ic-BL detected in untargeted surveys. We require that the classification of the $\mathrm{SNe}$ is robust. For example, we do not consider PTF12gzk, a SN that shows high expansion velocities (similar to SNe Ic-BL), yet its spectra do not show the persistent broad lines that are typical for Ic-BL SNe (Ben-Ami et al. 2012) and the automatic classification of Modjaz et al. (2014) classifies it as a SN Ic. We limit our comparison to events occurring at $z<0.2$, in order to have a similar redshift distribution of the SNe Ic-BL with and without a GRB. Since the southern part of the sky has not been covered by deep optical surveys at the time of our study, we limit our sample to declinations $\delta>-30^{\circ}$. Twenty-nine SNe Ic-BL satisfy our selection criteria. However, the host of SN2014ad lies near a very bright star whose contamination in the images of the host is very hard to properly account for. Therefore we do not consider this one in the further analysis and we are left with a sample of 28 SNe Ic-BL. Eight spectroscopically confirmed GRB-SNe have been detected up to this redshift. The two samples are summarised in Table 1.

Our main resource of photometric images are deep all-sky surveys. In particular, we use the Sloan Digital Sky Survey (SDSS) Data Release 12 (Alam et al. 2015) and PanSTARRS Data Release 1 (Chambers et al. 2016) data. For GRB-SNe, deep exposures obtained via GRB follow-up studies are available. We collected the available images obtained with various facilities. We limit our imaging analysis to the data taken with the Sloan $r$ filter (in the case of SDSS and PanSTARRS data) or to the images taken with filters with a similar wavelength range. The images of the galaxies in the sample are given in Fig. 1. The detailed account is provided in Table 1 . We search for the most accurate positions of detected $\mathrm{SNe}$ in the papers and, if those are not available, in the discovery telegrams. We note that for $\mathrm{SNe}$ Ic-BL, the positions are quoted without uncertainty in the

\footnotetext{
https://sne.space/
} 
Table 1. Samples of SNe Ic-BL without (28) and with (8) a GRB studied in this work.

\begin{tabular}{|c|c|c|c|c|c|}
\hline $\mathrm{SN} / \mathrm{GRB}$ & Redshift & Image & $\begin{array}{c}\mathrm{RA}_{\mathrm{SN}} \\
\text { (hh:mm:ss) }\end{array}$ & $\begin{array}{c}\operatorname{Dec}_{\mathrm{SN}} \\
\text { (dd:mm:ss) }\end{array}$ & Ref. \\
\hline SN2005ks & 0.0988 & SDSS-r & $21: 37: 56.56$ & $-00: 01: 56.90$ & Zheng et al. (2008) \\
\hline SN2005nb & 0.024 & SDSS-r & $12: 13: 37.61$ & $+16: 07: 16.20$ & Quimby et al. (2006) \\
\hline SDSS-IISN14475 & 0.1440 & SDSS-r & $22: 24: 30.96$ & $+00: 12: 12.28$ & Sako et al. (2018) \\
\hline SN2006nx & 0.125 & SDSS-r & $03: 33: 30.63$ & $-00: 40: 38.24$ & Bassett et al. (2006) \\
\hline SN2007bg & 0.034 & SDSS-r & $11: 49: 26.18$ & $+51: 49: 21.8$ & Moretti et al. (2007) \\
\hline SN2007ce & 0.046 & SDSS-r & 12:10:17.96 & $+48: 43: 31.51$ & Quimby et al. (2007) \\
\hline SN2007I & 0.0216 & SDSS-r & $11: 59: 13.15$ & $-01: 36: 18.9$ & Jin et al. (2007) \\
\hline PTF10aavz & 0.062 & SDSS-r & $11: 20: 13.36$ & $+03: 44: 45.20$ & Arcavi et al. (2010) \\
\hline SN2010ah & 0.0498 & SDSS-r & $11: 44: 02.99$ & $+55: 41: 27.60$ & Ofek et al. (2010) \\
\hline SN2010ay & 0.0671 & SDSS-r & $12: 35: 27.19$ & $+27: 04: 02.78$ & Drake et al. (2010) \\
\hline PTF10qts & 0.0907 & SDSS-r & $16: 41: 37.60$ & $+28: 58: 21.10$ & Gal-Yam et al. (2010) \\
\hline PTF10vgv & 0.015 & SDSS-r & $22: 16: 01.17$ & $+40: 52: 03.30$ & Corsi et al. (2012) \\
\hline PTF10xem & 0.05 & SDSS-r & 01:47:06.88 & $+13: 56$ & Corsi et al. (2016) \\
\hline PTF $11 \mathrm{cmh}$ & 0.1055 & SDSS-r & $13: 10: 21.74$ & $+37: 52: 59.60$ & Corsi et al. (2016) \\
\hline PTF11img & 0.158 & SDSS-r & $17: 34: 36.30$ & $+60: 48: 50.60$ & Corsi et al. (2016) \\
\hline PTF11lbm & 0.039 & SDSS-r & $23: 48: 03.20$ & $+26: 44: 33.50$ & Corsi et al. (2016) \\
\hline PTF12as & 0.033 & SDSS-r & 10:01:34.05 & $+00: 26: 58.40$ & Corsi et al. (2016) \\
\hline PTF13alq & 0.054 & SDSS-r & $11: 48: 02.03$ & $+54: 34: 38.60$ & The Open Supernova Catalog \\
\hline PTF13ebw & 0.069 & SDSS-r & $08: 17: 15.88$ & $+56: 34: 41.60$ & Corsi et al. (2016) \\
\hline PTF13u & 0.1 & SDSS-r & $15: 58: 51.21$ & $+18: 13: 53.10$ & Corsi et al. (2016) \\
\hline LSQ14bef & 0.05 & PanSTARRS-r & $14: 27: 41.77$ & $-08: 36: 45.50$ & Polshaw et al. (2014) \\
\hline PTF14dby & 0.074 & & $15: 17: 06.29$ & $+25: 21:$ & Prentice et al. (2016) \\
\hline PTF14gaq & 0.0826 & SDSS-r & $21: 32: 54.08$ & $+17: 44: 35.60$ & Corsi et al. (2016) \\
\hline iPTF15dld & 0.047 & SDSS-r & $00: 58: 13.28$ & $-03: 39: 50.29$ & Singer et al. (2015) \\
\hline SN2016coi & 0.003646 & SDSS-r & $21: 59: 04.12$ & $+18: 11: 10.72$ & Gaia Alerts ${ }^{a}$ \\
\hline SN2016dst & 0.074 & PanSTARRS-r & $04: 55: 54.68$ & $-29: 34: 13.73$ & Gaia Alerts \\
\hline SN2017dcc & 0.0245 & PanSTARRS-r & $12: 49: 04.89$ & $-12: 12: 22.57$ & Gaia Alerts \\
\hline SN2017fgk & 0.034 & PanSTARRS-r & $17: 47: 49.19$ & $+16: 08: 05.18$ & Transient Name Server $^{b}$ \\
\hline GRB 980425 & 0.0088 & VLT/FORS2-R & $19: 35: 03.31$ & $-52: 50: 44.7$ & Tinney et al. (1998) \\
\hline GRB 030329 & 0.1685 & HST/ACS-F606W & $10: 44: 49.96$ & $+21: 31: 17.44$ & Taylor et al. (2004) \\
\hline GRB 031203A & 0.1055 & VLT/FORS2-R & 08:02:30.16 & $-39: 51: 03.51$ & Soderberg et al. (2004) \\
\hline GRB 060218 & 0.03302 & HST/ACS-F625W & $03: 21: 39.68$ & $+16: 52: 01.82$ & Soderberg (2007) \\
\hline GRB 100316D & 0.0593 & HST/WCS-F625W & $07: 10: 30.56$ & $-56: 15: 20.18$ & Starling et al. (2011) \\
\hline GRB 130702A & 0.145 & VLT/FORS2-R & $14: 29: 14.77$ & $+15: 46: 26.37$ & Singer et al. (2013) \\
\hline GRB 161219B & 0.1475 & PanSTARRS-r & $06: 06: 51.42$ & $-26: 47: 29.52$ & Alexander et al. (2016) \\
\hline GRB 171205A & 0.0368 & PanSTARRS-r & 11:09:39.52 & $-12: 35: 18.48$ & Laskar et al. (2017) \\
\hline
\end{tabular}

Notes. For each host galaxy, we report: redshift, survey or instrument (and filter) of the images that were analysed, and the position at which a SN occurred. References for positions are given in the last column. Images of hosts of GRB-SNe, which are not part of all-sky surveys, were retrieved from: GRB 980425 (ESO archive, Sollerman et al. 2005), GRB 030329 (HST archive, Sollerman et al. 2005), GRB 031203A (ESO archive, Mazzali et al. 2006), GRB 060218 (HST archive, PI: S.R. Kulkarni), GRB 100316D (HST archive, Cano et al. 2011), GRB 130702A (ESO archive, PI: E. Pian). ${ }^{(a)}$ http://gsaweb.ast.cam.ac.uk/alerts, ${ }^{(b)}$ https://wis-tns. weizmann.ac.il/.

majority of cases. We discuss how this impacts our results in Sect. 3.2. The SN position coincides with a galaxy in nearly all the cases in our sample. In two cases we could not carry out all the measurements either because a galaxy is not detected (PTF11img) or because the galaxy is detected but too faint (SN2007bg) - we discuss the implications for the results later on. Several cases that merit special attention are discussed in Sect. 2.2.

\subsection{Measuring luminosities and sizes}

The main parameters that we are interested in are galaxy luminosity, their (physical) size, and the position of the SN site relative to the host galaxy centre (projected offset, in the following: "offset"). Galaxies in the sample span a large interval of apparent sizes, magnitudes, and morphologies. The images that we analyse come from different instruments. Taking that into account, one has to make some compromises with the analysis (i.e. sometimes one technique gives a more reliable result than another). All galaxies in the sample have been analysed using SExtractor (v2.19.5; Bertin \& Arnouts 1996). For the measured apparent magnitudes, we adopt the MAG_AUTO magnitudes, corresponding to the flux within 2.5 Kron (1980) radii. With SExtractor, we can also obtain the measurements of $r_{50}$ and $r_{90}$, representing the radii within which 50 and $90 \%$ of the light is enclosed, respectively. However, many of the galaxies in the sample have a very small apparent size, comparable to the size of the point-spread function (PSF) of the image. To take the PSF accurately into account, we measure galaxy sizes by fitting their surface brightness profiles with a simple parametric model using GALFIT (Peng et al. 2010). We generate PSFs of 

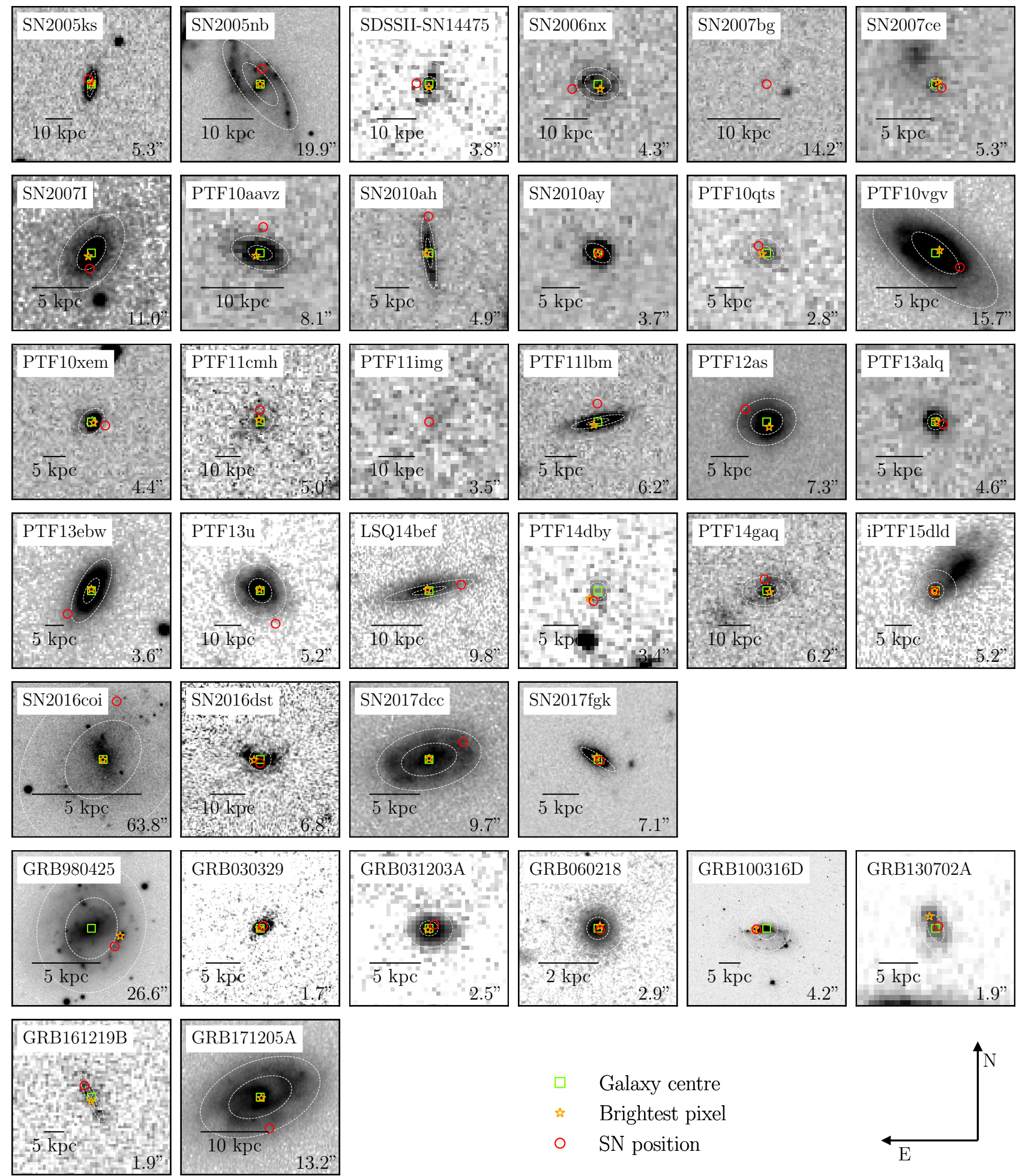

Fig. 1. Overview of the galaxies in the sample. See Table 1 for the information on the survey or the instrument with which each image has been taken. The red circle indicates the position of each SN: the size of the circle is arbitrary. The green square and the orange star indicate the measured galaxy centre and the brightest pixel in the galaxy, respectively. Overplotted are ellipses corresponding to the measured $r_{50}$ and $r_{90}$ radii, where the values of position angle and inclination (see Table A.1) have been used to take geometric effects into account. The values provided in the bottom right corners correspond to the physical scales in each figure. All images are centred on galaxy centres except for the case of SN2016coi. We note that in the case of PTF11img the host galaxy is not detected.

individual images using the PSFEx (Bertin 2011), a routine that together with SExtractor extracts models of the PSF at desired positions in the images. This PSF is then used as an input of the GALFIT fitting procedure. For simplicity, we fit each galaxy with one Sersic profile and, if statistically justified, with an additional exponential profile with a common centre. Most of the galaxies in the sample are well described with this simple model. The exception are galaxies which are well resolved and 
Table 2. Results of the analysis of the host galaxies.

\begin{tabular}{|c|c|c|c|c|c|c|c|c|}
\hline SN/GRB & $\mathrm{RA}_{\text {host }}$ & Dec ${ }_{\text {host }}$ & $\begin{array}{c}m_{\mathrm{R}} \\
(\mathrm{mag})\end{array}$ & $\begin{array}{c}M_{\mathrm{R} /(1+\mathrm{z})} \\
(\mathrm{mag})\end{array}$ & $\begin{array}{c}r_{50} \\
(\mathrm{kpc})\end{array}$ & $\begin{array}{c}r_{90} \\
(\mathrm{kpc})\end{array}$ & $\begin{array}{l}\text { Offset }_{\text {centre }} \\
\quad(\mathrm{kpc})\end{array}$ & $\begin{array}{r}\text { Offset }_{\mathrm{bp}} \\
(\mathrm{kpc})\end{array}$ \\
\hline SN2005ks & $21: 37: 56.54$ & $-00: 01: 57.84$ & $18.48 \pm 0.17$ & $-19.92 \pm 0.17$ & $2.26 \pm 0.09$ & $4.76 \pm 0.19$ & 1.88 & 1.74 \\
\hline $\mathrm{SN} 2005 \mathrm{nb}^{a}$ & $12: 13: 37.65$ & $+16: 07: 10.29$ & $14.66 \pm 0.01$ & $-20.58 \pm 0.01$ & 5.13 & 10.84 & 2.99 & 2.97 \\
\hline SDSS-IISN14475 & $22: 24: 30.87$ & $+00: 12: 12.22$ & $20.93 \pm 0.12$ & $-18.36 \pm 0.12$ & $1.97 \pm 0.21$ & $5.59 \pm 0.71$ & 3.54 & 3.33 \\
\hline SN2006nx & $03: 33: 30.47$ & $-00: 40: 37.81$ & $19.96 \pm 0.06$ & $-19.11 \pm 0.06$ & $3.00 \pm 0.14$ & $5.07 \pm 0.37$ & 5.80 & 6.22 \\
\hline SN2007bg & $11: 49: 26.24$ & $+51: 49: 22.71$ & $>22.4$ & $>-13.6$ & & & & \\
\hline \multirow[t]{2}{*}{$\mathrm{SN} 2007 \mathrm{ce}^{b}$} & 12:10:18.02 & $+48: 43: 31.87$ & $20.85 \pm 0.24$ & $-15.78 \pm 0.24$ & $0.34 \pm 0.02$ & $0.65 \pm 0.23$ & 0.65 & $0.63^{c}$ \\
\hline & $12: 10: 18.19$ & $+48: 43: 34.69$ & $19.33 \pm 0.14$ & $-17.30 \pm 0.14$ & $2.48 \pm 0.24$ & $5.10 \pm 0.67$ & 3.67 & \\
\hline SN2007I & 11:59:13.13 & $-01: 36: 15.88$ & $17.40 \pm 0.02$ & $-17.59 \pm 0.02$ & $1.79 \pm 0.03$ & $4.08 \pm 0.08$ & 1.38 & 1.09 \\
\hline PTF10aavz & $11: 20: 13.38$ & $+03: 44: 42.63$ & $19.24 \pm 0.04$ & $-18.13 \pm 0.04$ & $1.49 \pm 0.07$ & $3.51 \pm 0.21$ & 3.17 & 3.55 \\
\hline SN2010ah & $11: 44: 02.98$ & $+55: 41: 22.34$ & $19.07 \pm 0.18$ & $-17.71 \pm 0.18$ & $2.21 \pm 0.06$ & $5.21 \pm 0.16$ & 5.32 & 5.34 \\
\hline SN2010ay & $12: 35: 27.20$ & $+27: 04: 02.76$ & $19.05 \pm 0.03$ & $-18.40 \pm 0.30$ & $0.33 \pm 0.01$ & $1.07 \pm 0.16$ & 0.17 & 0.25 \\
\hline PTF10qts & $16: 41: 37.53$ & $+28: 58: 20.36$ & $21.23 \pm 0.21$ & $-16.91 \pm 0.21$ & $1.28 \pm 0.26$ & $3.07 \pm 0.81$ & 2.07 & 1.51 \\
\hline PTF10vgv & 22:16:01.59 & $+40: 52: 06.07$ & $15.29 \pm 0.04$ & $-19.21 \pm 0.04$ & $1.81 \pm 0.05$ & $4.75 \pm 0.19$ & 1.75 & 1.64 \\
\hline PTF10xem & 01:47:07.06 & $+13: 56: 29.48$ & $18.60 \pm 0.03$ & $-18.57 \pm 0.03$ & $1.26 \pm 0.06$ & $3.75 \pm 0.17$ & 3.18 & 2.66 \\
\hline PTF11 cmh & $13: 10: 21.73$ & $+37: 52: 57.33$ & $19.89 \pm 0.33$ & $-18.55 \pm 0.33$ & $5.79 \pm 0.56$ & $12.25 \pm 1.59$ & 4.54 & 3.84 \\
\hline PTF11img & & & $>22.5$ & $>-16.9$ & & & & \\
\hline PTF11lbm & 23:48:03.18 & $+26: 44: 30.05$ & $17.95 \pm 0.03$ & $-18.42 \pm 0.03$ & $1.79 \pm 0.06$ & $4.18 \pm 0.22$ & 3.62 & 3.36 \\
\hline PTF12as & $10: 01: 33.78$ & $+00: 26: 56.04$ & $16.47 \pm 0.01$ & $-19.44 \pm 0.01$ & $2.14 \pm 0.01$ & $3.95 \pm 0.03$ & 3.25 & 3.90 \\
\hline PTF13alq & 11:48:02.12 & $+54: 34: 38.87$ & $19.97 \pm 0.10$ & $-17.00 \pm 0.10$ & $0.26 \pm 0.04$ & $0.84 \pm 0.04$ & 0.86 & 0.74 \\
\hline PTF13ebw & $08: 17: 15.32$ & $+56: 34: 46.12$ & $16.27 \pm 0.06$ & $-21.35 \pm 0.06$ & $3.66 \pm 0.19$ & $9.33 \pm 0.74$ & 8.83 & 9.08 \\
\hline PTF13u & 15:58:51.42 & $+18: 13: 59.60$ & $17.02 \pm 0.02$ & $-21.38 \pm 0.02$ & $4.83 \pm 0.10$ & $10.84 \pm 0.21$ & 13.56 & 14.0 \\
\hline LSQ14bef & $14: 27: 42.24$ & $-08: 36: 46.45$ & $17.52 \pm 0.02$ & $-19.35 \pm 0.01$ & $3.34 \pm 0.03$ & $8.94 \pm 0.80$ & 7.14 & 6.69 \\
\hline PTF14dby & $15: 17: 06.28$ & $+25: 21: 11.90$ & $22.14 \pm 0.08$ & $-15.61 \pm 0.08$ & $1.23 \pm 0.23$ & $1.91 \pm 0.35$ & 0.76 & 0.69 \\
\hline PTF14gaq & $21: 32: 54.05$ & $+17: 44: 33.50$ & $19.33 \pm 0.20$ & $-18.80 \pm 0.20$ & $3.50 \pm 0.25$ & $7.66 \pm 0.58$ & 3.62 & 4.30 \\
\hline \multirow[t]{2}{*}{ iPTF15dld $^{b}$} & $00: 58: 13.27$ & $-03: 39: 50.14$ & $18.27 \pm 0.12$ & $-18.43 \pm 0.12$ & $1.76 \pm 0.07$ & $4.07 \pm 0.24$ & 0.20 & $0.12^{c}$ \\
\hline & 00:58:12.95 & $-03: 39: 46.15$ & $16.67 \pm 0.07$ & $-20.03 \pm 0.07$ & $3.49 \pm 0.14$ & $7.57 \pm 0.32$ & 6.17 & \\
\hline SN2016dst & $04: 55: 54.68$ & $-29: 34: 12.99$ & $18.55 \pm 0.04$ & $-19.11 \pm 0.04$ & $3.07 \pm 0.07$ & $6.33 \pm 0.17$ & 1.08 & 1.94 \\
\hline SN2016coi & 21:59:04.69 & $+18: 10: 36.67$ & $12.95 \pm 0.06$ & $-18.30 \pm 0.06$ & $2.00 \pm 0.16$ & $4.41 \pm 0.40$ & 2.70 & 2.72 \\
\hline SN2017dcc & $12: 49: 05.34$ & $-12: 12: 25.91$ & $15.90 \pm 0.01$ & $-19.40 \pm 0.01$ & $2.79 \pm 0.02$ & $5.27 \pm 0.07$ & 3.72 & 3.77 \\
\hline SN2017fgk & $17: 47: 49.25$ & $+16: 08: 05.79$ & $17.33 \pm 0.03$ & $-18.81 \pm 0.03$ & $1.08 \pm 0.03$ & $2.80 \pm 0.07$ & 0.75 & 0.62 \\
\hline GRB 980425 & $19: 35: 04.00$ & $-52: 50: 37.68$ & $14.37 \pm 0.05$ & $-18.66 \pm 0.05$ & $2.25 \pm 0.03$ & $4.74 \pm 0.03$ & 2.25 & 0.87 \\
\hline GRB 030329 & $10: 44: 49.94$ & $+21: 31: 17.35$ & $23.09 \pm 0.02$ & $-16.42 \pm 0.02$ & $0.54 \pm 0.03$ & $1.37 \pm 0.08$ & 0.86 & 0.59 \\
\hline GRB 031203A & 08:02:30.18 & $-39: 51: 03.68$ & $20.65 \pm 0.03$ & $-20.19 \pm 0.03$ & $1.04 \pm 0.04$ & $2.79 \pm 0.16$ & 0.34 & 0.64 \\
\hline GRB 060218 & 03:21:39.69 & 16:52:01.88 & $20.30 \pm 0.06$ & $-15.88 \pm 0.06$ & $0.37 \pm 0.01$ & $0.85 \pm 0.05$ & 0.08 & 0.12 \\
\hline GRB 100316D & $07: 10: 30.31$ & $-56: 15: 20.21$ & $17.80 \pm 0.05$ & $-19.61 \pm 0.05$ & $2.55 \pm 0.02$ & $5.42 \pm 0.10$ & 2.48 & 0.39 \\
\hline GRB 130702A & $14: 29: 14.78$ & $+15: 46: 26.25$ & $23.34 \pm 0.12$ & $-15.88 \pm 0.12$ & $1.85 \pm 0.16$ & $3.77 \pm 0.30$ & 0.49 & 1.64 \\
\hline GRB 161219B & 06:06:51.37 & $-26: 47: 30.62$ & $20.67 \pm 0.03$ & $-18.56 \pm 0.03$ & $3.19 \pm 0.16$ & $7.17 \pm 0.62$ & 3.45 & 4.37 \\
\hline GRB 171205A & 11:09:39.69 & $-12: 35: 11.31$ & $15.33 \pm 0.02$ & $-20.90 \pm 0.02$ & $4.86 \pm 0.03$ & $9.25 \pm 0.06$ & 5.77 & 5.58 \\
\hline
\end{tabular}

Notes. Columns are: barycentre position $\left(\mathrm{RA}_{\text {host }}, \mathrm{Dec}_{\text {host }}\right.$ ) of the host galaxy, apparent and absolute magnitude, radii $r_{50}$ and $r_{90}$ (radii containing 50

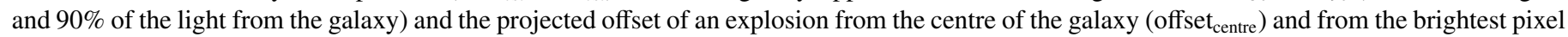
( offset $_{\mathrm{bp}}$ ). Absolute magnitudes are corrected for Galactic foreground extinction (Schlafly \& Finkbeiner 2011), while the apparent magnitudes are not. Systematic errors on measured offsets are discussed in Sect. 3.2. ${ }^{(a)}$ The surface brightness of this host can not be modelled with a simple phenomenological model, therefore the results are from photometric analysis and the measured characteristic radii are without errors. ${ }^{(b)}$ The first line corresponds to the measurement assuming the host galaxy is only the bright (blue) region near the SN explosion. The second line is obtained in the case the host galaxy is the whole complex. ${ }^{(c)}$ The brightest pixel is located in the compact region of the complex.

very big on the sky, where the simple model cannot account for the presence of features like spiral arms. In these few cases (GRB980425, SN2005nb, SN2016coi), the PSF does not play an important role and we adopt the SExtractor measurements. We note that the magnitudes measured from the GALFIT analysis are consistent with those obtained with SExtractor. Measured values of $r_{50}$ are consistently smaller with the GALFIT for objects with $r_{50} \lesssim F W H M$ (PSF), which is exactly what we expect.

With SExtractor, we estimate the barycentre of each galaxy (simply "centre" in the following) and the brightest pixel in each galaxy (see e.g. Lyman et al. 2017). Finally, we measure the offset between the SN position and the centre of their host galaxy. The measured positions - galaxy centre, the position of the brightest pixel, and the position of the detected SN explosion - are indicated in Fig. 1 for each individual case.

The measured $r_{50}$ and $r_{90}$ radii are transformed to proper sizes and the magnitude is transformed to absolute magnitude as $M_{\mathrm{R} /(1+z)}=m_{\mathrm{R}}-A_{\mathrm{R}}-5 \log \left(D_{\mathrm{L}} / 10 \mathrm{pc}\right)+2.5 \log (1+z)$, where $A_{\mathrm{R}}$ is the Galactic foreground extinction taken from the maps of Schlafly \& Finkbeiner (2011). All the measurements are collected in Table 2. 


\subsection{Individual host galaxies}

SN2007bg. There is a very faint source near the position of the $\mathrm{SN}$, which we assume to be the actual host galaxy. The other two sources nearby are offset by such an amount that the projected offsets, normalized by the size of the galaxy (i.e. $r_{50}$ ), when computed, are in a completely different regime to the rest of the sample. The presumable host is too faint to allow for a reliable magnitude and size measurement. We only provide a magnitude upper limit and an estimate of the projected offset. We note that Kelly \& Kirshner (2012) presumably assumed the nearby bright source to be the host galaxy. However, if we make the same assumption, we measure a larger normalized projected offset as the one provided by Kelly \& Kirshner (2012).

SN2007ce. The SN occurred near the position of a compact, blue region. It is unclear whether or not the region is a part of the larger complex (see Fig. 1). We conduct the analysis for both scenarios (see Table 2). For subsequent analyses, we conservatively assume that the compact region is a galaxy on its own.

iPTF15dld. The SN occurred at the position of a bright, compact starburst region (see Fig. 1). It is unclear whether or not the region is a part of the whole system (see also Pian et al. 2017). We conduct the analysis for both scenarios (see Table 2). For subsequent analyses, we conservatively assume that the compact region is a galaxy on its own. In the alternative scenario the $\mathrm{SN}$ is found at a much larger normalized offset.

SN2005ks. The reported position of the SN differs in Zheng et al. (2008) and Sako et al. (2018), even though the position in both works is determined from the SDSS detection. We adopt the position determined by Zheng et al. (2008). This position places the SN about half as far from the host's centre as does that of Sako et al. (2018).

PTFllimg. The host galaxy is not detected in the SDSS image. We only derive a magnitude upper limit.

$G R B$ 161219B. An image of the host has been taken with the HST/WFC3 camera using the F200LP filter (Cano et al. 2017a). Because this filter covers a much broader wavelength range compared to the other filters in this study, we prefer to use the PanSTARRS image for the analysis. We note that the host is much better resolved in the HST image. The analysis of the HST image results in very similar offsets and slightly larger sizes $\left(r_{50}=3.75 \pm 0.08 \mathrm{kpc}\right)$ compared to the values measured from the PanSTARRS image.

\section{Results}

We compare the distributions of the studied characteristics of the two samples: galaxy size $r_{50}$ and $r_{90}$ and the offsets of SN location with respect to the galaxy centre and the brightest pixel. For each distribution we compute the median and $1 \sigma$-equivalent confidence interval - we do not take the errors in the computation of these statistics into account. We use the two-sided AndersonDarling (AD) test between any two distributions to estimate a chance probability that the two distributions of a given characteristic are drawn from the same parent distribution. The errors are taken into account by performing a Monte Carlo (MC) simulation: we perform the AD test 10000 times on the distributions built by randomly varying the measured characteristic for its error. The results are summarised in Table 3.

\subsection{Absolute magnitude and size}

The two samples have a similar absolute magnitude distribution (Fig. 3a), especially when considering the two non-detections.
Table 3. Statistical description of the data of the SN Ic-BL and GRB-SN samples.

\begin{tabular}{lllllll}
\hline \hline & \multicolumn{3}{c}{ Median $( \pm 1 \sigma)$} & & \multicolumn{2}{c}{ AD test } \\
\cline { 2 - 3 } Characteristic & SN Ic-BL & GRB-SN & & $p_{\text {AD }}$ & $p_{\text {AD }, \mathrm{MC}}$ \\
\hline$r_{50}(\mathrm{kpc})$ & $1.99_{-1.05}^{+1.90}$ & $2.05_{-1.60}^{+1.94}$ & & 0.87 & $0.87_{-0.07}^{+0.11}$ \\
$r_{90}(\mathrm{kpc})$ & $4.58_{-2.83}^{+5.04}$ & $4.26_{-3.16}^{+4.02}$ & & 0.87 & $0.90_{-0.15}^{+0.13}$ \\
Offset $_{\text {centre }}(\mathrm{kpc})$ & $3.08_{-2.35}^{+2.98}$ & $1.54_{-1.28}^{+3.13}$ & & 0.15 & $0.18_{-0.13}^{+0.16}$ \\
Offset $_{\mathrm{bp}}(\mathrm{kpc})$ & $3.15_{-2.52}^{+3.16}$ & $0.76_{-0.51}^{+4.26}$ & & 0.10 & $0.07_{-0.04}^{+0.09}$ \\
Offset $_{\text {centre }} / \mathrm{r}_{50}$ & $1.43_{-0.86}^{+0.99}$ & $0.98_{-0.74}^{+0.43}$ & & 0.07 & $0.07_{-0.04}^{+0.07}$ \\
Offset $_{\mathrm{bp}} / \mathrm{r}_{50}$ & $1.52_{-0.92}^{+1.03}$ & $0.75_{-0.52}^{+0.54}$ & & 0.02 & $0.02_{-0.01}^{+0.04}$ \\
\hline
\end{tabular}

Notes. Reported are the median and $1 \sigma$-equivalent values of the distributions of several galaxy characteristics of our samples. The last two columns give the results of the two-sided Anderson-Darling test for comparing the distributions of the corresponding characteristics of the two samples. AD chance probabilities are given for the case when distributions are taken at face values $\left(p_{\mathrm{AD}}\right)$ and when errors and systematics are taken into account $\left(p_{\mathrm{AD}, \mathrm{MC}}\right)$.

Without the non-detections, the two-sided AD test between the two distributions gives a chance probability of $p_{\mathrm{ch}}=0.72$ that the two distributions are drawn from the same parent distribution. In calculating absolute magnitudes we did not take into account the fact that the galaxies are at different redshifts and that they are observed with different filters. Given the redshift range and typical spectral shapes, the different filter characteristics are expected to introduce uncertainties at the level of a few to $\sim 10 \%$. Nevertheless, as there are many uncertainties involved in the comparison of absolute magnitudes, the results should be taken with caution and we do not include a detailed statistical summary in Table 3.

The galaxies in the two samples have on average similar sizes when considering both $r_{50}$ and $r_{90}$ (Fig. 3). This is confirmed by performing an AD test (see Table 3). Many studies of long GRB host galaxy samples have taken similar measurements as presented in this work, mostly using HST images for the measurements. Median sizes of long GRB hosts in the literature (typically covering samples in large redshift ranges and measured on images taken at different rest-frame wavelengths) are $r_{50 \text {,med }}=1.8 \pm 0.1 \mathrm{kpc}$ (Blanchard et al. 2016), $1.7 \pm 0.2 \mathrm{kpc}$ (Lyman et al. 2017), and $1.5 \pm 0.5 \mathrm{kpc}$ (Bloom et al. 2002).

The redshift distribution of the GRB-SN sample is skewed slightly towards higher redshift with respect to the regular SN sample (Fig. 2). According to Shibuya et al. (2015), the average size evolution of star-forming galaxies can be approximated with the $(1+z)^{-1.2}$ relation. Using it at face value, the small difference in the average redshift of our distributions results in a negligible difference in the mean size. Any significant difference in the size between the two populations should therefore be attributed to the intrinsic difference of the populations.

At $z \gtrsim 0.05$, the $\mathrm{H} \alpha$ nebular emission line falls out of the filters with which the images analysed in this work have been taken. $\mathrm{H} \alpha$ can be quite strong in star forming galaxies and therefore it could have a (small) effect on our measurements of galaxy sizes and offsets. We check how this affects our results by analysing the $z>0.05$ part of the SN Ic-BL sample, this time analysing the $i$-band SDSS and PanSTARRS images. We find very good agreement between the sizes and offsets measured from $r$ - and $i$-band images. 


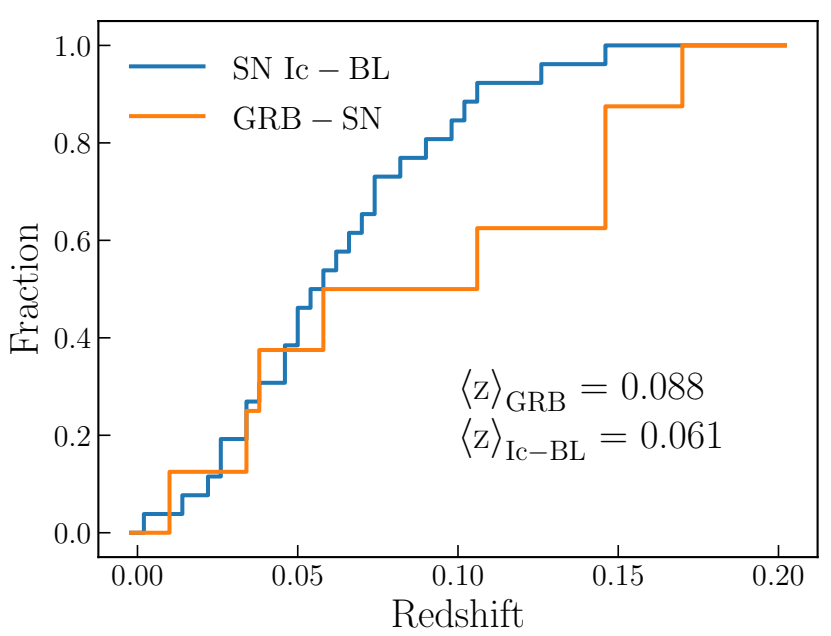

Fig. 2. Cumulative redshift distributions of the GRB-SN and SN Ic-BL samples. Indicated are mean redshifts of the distributions.

\subsection{Offsets}

Next we look at the offset of the explosion from the host galaxy centre. SNe Ic-BL with GRBs are found closer to their host galaxy centre on average (Fig. 4a). As the galaxies span a large range in size ( $r_{50} \sim 0.2-6 \mathrm{kpc}$ for both populations), a more relevant quantity to compare is the offset normalized by the galaxy size. We use the measured $r_{50}$ for normalization. The distributions for the two samples are shown in Fig. $4 \mathrm{~b}$ and they are clearly distinct, that is, GRB-SNe occur closer to their host galaxy centre than the SNe Ic-BL. This is confirmed by performing the $\mathrm{AD}$ test between the two distributions resulting in the chance probability of $p_{\mathrm{ch}}=0.07$ that the two distributions are drawn from the same parent distribution.

One can also look at the offsets from the brightest pixels. In general, the position of a galaxy's barycentre and its brightest pixel are similar for our samples (see Fig. 1 and Table 2). The difference between normalized offsets between $\mathrm{SN}$ positions and the brightest pixels in a galaxy is found to be even more significant $\left(p_{\mathrm{ch}}=0.02\right)$. However, we note that this difference arises mostly due to the case of GRB 980425, where the brightest pixel is not located near the galaxy's centre but instead in a well-known Wolf-Rayet region located $\approx 0.9 \mathrm{kpc}$ from the GRB (Hammer et al. 2006; Krühler et al. 2017).

The positions of SNe Ic-BL are typically reported without uncertainties. While five of the $\mathrm{SNe}$ discovered either by the SDSS survey (2) or the Gaia satellite (3) should be very accurate, we cannot claim that for the majority of the sample. The uncertainty in the SN position is expected to be much higher than the uncertainty in the astrometric calibration. We estimate the uncertainty of astrometric calibration of the SDSS and PanSTARRS images by comparing the positions of the relatively bright sources in the images to the catalogued values. The measured uncertainties $^{3}$ are $<0.1^{\prime \prime}$ in all cases. Given the sizes of the galaxies in the sky, and assuming the worst-case scenario in which the SN positions are uncertain by $\sim 1^{\prime \prime}$, then approximately $20 \%$ of the SN sample could be affected. In the case of GRB-SNe, the positions are well constrained $\left(\lessgtr 0.2^{\prime \prime}\right)$ due to the availability of radio detections of bright afterglows. The measured astrometric uncertainties in the VLT/FORS2 images as well as PanSTARRS are $<0.1^{\prime \prime}$. The HST image of GRB 030329 host was aligned

\footnotetext{
3 For typical astrometric uncertainties in the SDSS images (which agree with our estimates) see also Pier et al. (2003).
}
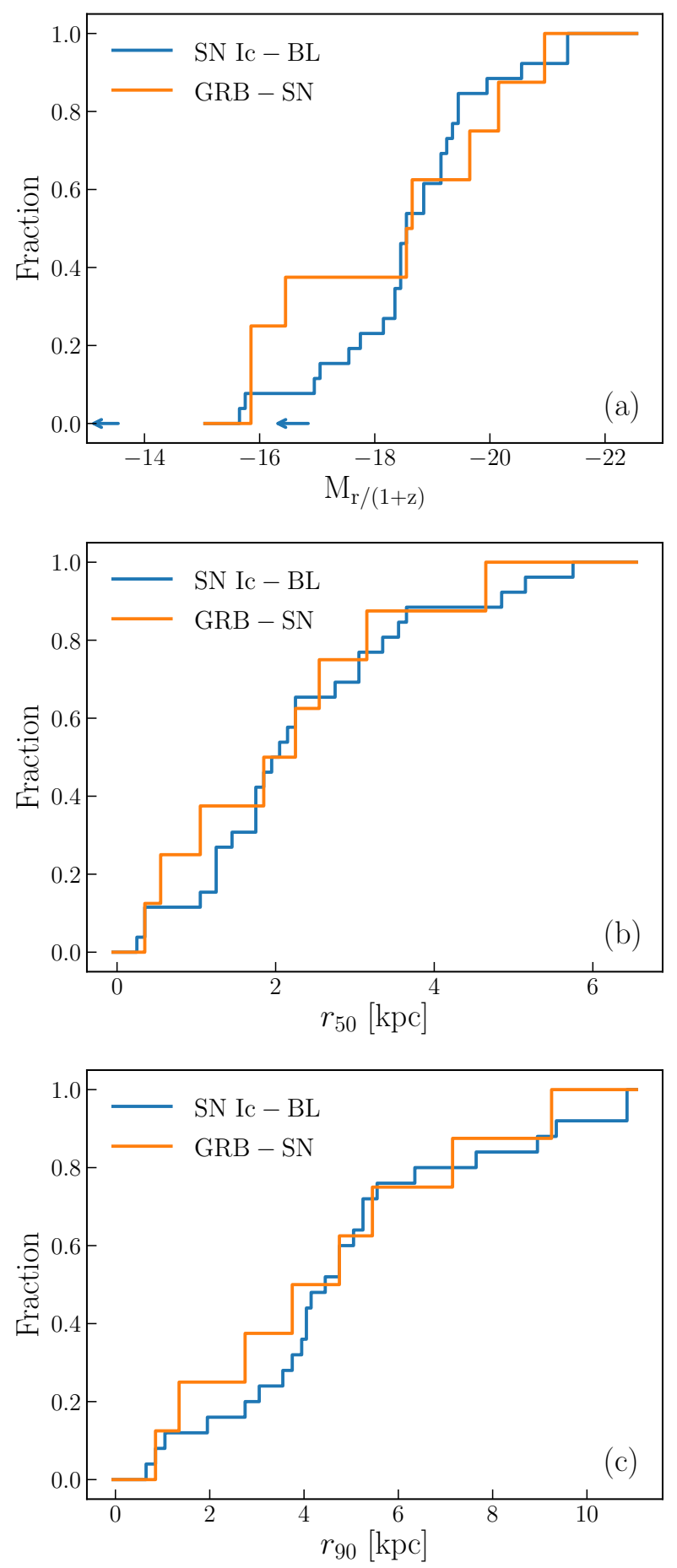

Fig. 3. Comparing cumulative distributions of panel a: luminosity; panel $b: r_{50}$ radii and panel $c: r_{90}$ radii of the GRB-SN and SN Ic-BL samples. The blue arrows in figure (panel a) correspond to the upper limits measured for the hosts of SN2007bg and PTF11img.

with an image of the afterglow taken in the same filter. The HST image of GRB 100316D host was aligned with an image of the afterglow taken in a similar (but not the same) filter. The estimated uncertainty for these two hosts is $<0.1^{\prime \prime}$. The astrometric calibration for the HST image of GRB 060218 was achieved by alignment with the calibrated VLT/FORS2 image of the same field and the estimated uncertainty is $\sim 0.2^{\prime \prime}$. Combining uncertainties in astrometric calibrations with uncertainties of the afterglow position, the uncertainties are $<0.2^{\prime \prime}$ in all cases studied in 

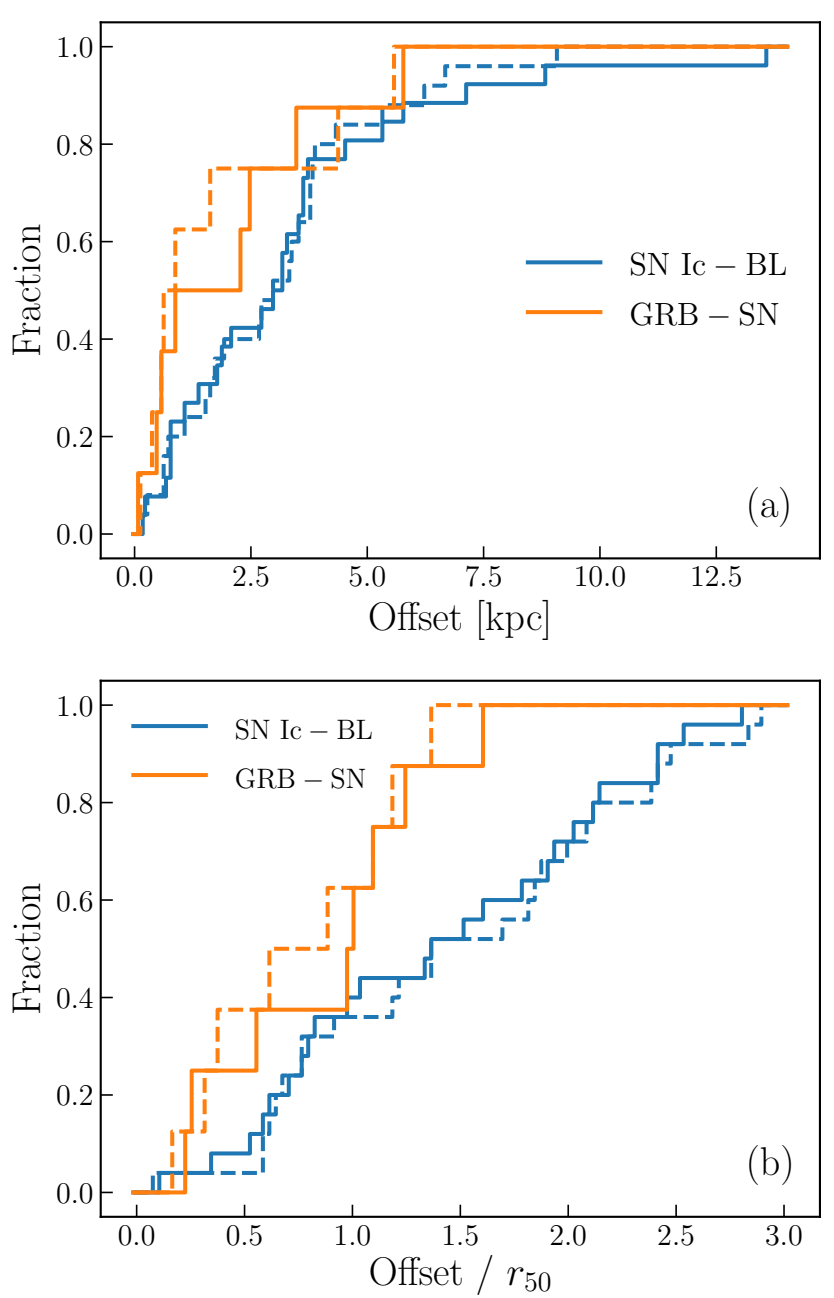

Fig. 4. Cumulative distributions of the projected offset (panel $a$ ) and the normalized offset (panel $b$ ) of the explosions from the host galaxy centre. Solid and dashed lines indicate offsets with respect to galaxy centres and the brightest pixel, respectively.

this paper. For the computation of the AD test using the MC simulation, we assume conservative systematic uncertainties of $0.5^{\prime \prime}$ and $0.2^{\prime \prime}$ for SNe Ic-BL and GRB-SNe, respectively. While the computed chance significance in this case has a distribution with a tail towards higher values, the results in Table 3 still indicate a difference.

Blanchard et al. (2016) find offset centre,med $=1.3 \pm 0.2 \mathrm{kpc}$ and offset centre $/ r_{50}=0.7 \pm 0.2$, in agreement with our results. Our results are also in agreement with Bloom et al. (2002) who find offset centre,med $=1.4 \pm 0.8 \mathrm{kpc}$ and offset centre $/ r_{50}=0.8 \pm 0.3$, while Lyman et al. (2017) finds offset centre,med $=1.0 \pm 0.2 \mathrm{kpc}$ and offset centre $/ r_{50}=0.6 \pm 0.1$.

In general, we do not expect the galaxies to have a preferential orientation and therefore, on statistical grounds, the fact that we are comparing projected offsets should not affect our results (other than introducing additional noise to the offset distributions). However, as our samples are small, that may not necessarily be the case. We therefore estimated the inclinations of our galaxies and calculated the deprojected offsets for the galaxies of our sample (see Appendix A). The analysis shows that the trend of GRB-SNe to be found closer to the centres of their host galaxies persists even when considering deprojected offsets.

The sample of GRB-SNe is small: using a bootstrap method by re-sampling, the chance probabilities have a long tail towards $p_{\text {ch }}>0.1$ (while the median changes only slightly). In addition, the sample of SNe Ic-BL could be contaminated by actual GRB$\mathrm{SNe}$ with a GRB jet being directed away from us. If one or two $\mathrm{SNe}$ Ic-BL with the high normalized offsets turn out to be GRB$\mathrm{SNe}$, the two distributions would become very similar (though the argument could also go in the other direction). Nevertheless, the results indicate that the two samples are drawn from different distributions, even if we cannot claim that with a high significance. With this in mind we therefore assume that the observed difference is real in the following discussion.

\section{Metallicities}

Since metallicity is considered to be one of the main parameters that influences the lives and fates of massive stars that are considered to be the progenitors of SNe and GRBs, we searched the literature for spectroscopic analyses of the hosts included in this paper in order to measure gas-phase metallicities from nebular emission lines. Modjaz et al. (2011) and Sanders et al. (2012) analyzed eight SNe Ic-BL hosts that are part of our sample. Additionally, we consider six $\mathrm{SNe}$ Ic-BL hosts from Modjaz et al. (2011) that were detected in a private untargeted survey, but whose classification is hard to confirm. We include these hosts in order to increase the sample - as shown below, this does not affect the results. For GRB-SNe, we can determine the metallicities for six hosts: GRB 980425 (Krühler et al. 2017), GRB 030329 (Gorosabel et al. 2005), GRB 031203 (Guseva et al. 2011), GRB 060218 (Wiersema et al. 2007), GRB 100316D (Izzo et al. 2017), GRB 161219B (Cano et al. 2017a). All the host galaxies are listed in Table 4. For comparison, we also collect spectral data for SN Ib and Ic host samples provided by Sanders et al. (2012) and Modjaz et al. (2011).

Given the availability of the detected lines, we compute the metallicities using two different diagnostics. The first one is the O3N2 diagnostic provided by Pettini \& Pagel 2004, where the metallicity is computed from the $[\mathrm{OIII}] \lambda 5007 / \mathrm{H}_{\beta}$ and [NII] $\lambda 6584 / \mathrm{H} \alpha$ ratios. In a few cases, where this diagnostic could not be used, we compute the metallicity using one of the other common calibrations and then transform its value to the O3N2 scale (Kewley \& Ellison 2008). The other diagnostic is the one provided by Maiolino et al. (2008), where the metallicity is obtained using as many different calibrations as possible and then minimising the probability density distribution (see also Japelj et al. 2016a,b for details). This diagnostic is very similar to the calibration provided by Kewley \& Dopita (2002). While Modjaz et al. (2011) does not provide spectral line measurements, they already compute the metallicities in both O3N2 and Kewley \& Dopita (2002) scales, which we adopt directly in this paper. All the values and references to the source material are gathered in Table 4.

The metallicity distributions are plotted in Fig. 5. For comparison, we also plot the metallicity distribution of the combined $z<1$ host galaxy complete BAT6 sample of GRBs (Salvaterra et al. 2012; Japelj et al. 2016a) and the sample of $z<1$ host galaxies from Krühler et al. (2015). It has been shown that the metallicity distribution of GRB host galaxies does not show any evolution at least up to $z \sim 2$ (Krühler et al. 2015), therefore the fact that the long GRB $z<1$ sample covers a different redshift regime is not problematic. Indeed, our GRB-SNe host galaxies have similar metallicities to the long GRB sample (we note that the two samples do not have any galaxies in common). On the other hand, SN Ic-BL host galaxies have metallicities skewed towards higher values. It is rather striking that 
$\sim 30 \%$ of the SNe Ic-BL are found to have super-solar metallicities. This is a much higher fraction than what is found for GRB host galaxies ( 10\%; Krühler et al. 2015; Japelj et al. 2016a). The difference in metallicities is observed both in the $\mathrm{O} 3 \mathrm{~N} 2$ and the Maiolino et al. (2008) diagnostics. We also note that for the $\mathrm{SNe}$ Ic-BL sample, the resulting distribution with and without adding the additional objects from Modjaz et al. (2011) is the same.

The metallicity distributions in Fig. 5 reveal that SN Ib and Ic-BL hosts have similar metallicity distributions (though the metallicities for SN Ic-BL hosts do extend to lower values). SN Ic hosts clearly have higher metallicities on average (see also Modjaz et al. 2011; Sanders et al. 2012). On the other hand, long GRB host galaxies seem to have lower metallicities on average than other types (see also e.g. Graham \& Fruchter 2013). For the sake of completeness, we provide a summary of the results of the AD two-sided test for metallicity distributions of different explosions' environments in Table 5. Based on the test, we cannot rule out that the GRB-SNe are found in the galaxies with similar metallicities as the hosts of SNe Ic-BL. If the distribution of GRB-SNe indeed follows the distribution of long GRBs, then the difference becomes slightly more significant. It is interesting to see that the lack of low-metallicity Ib hosts leads to a much more significant difference to GRB-SNe and long GRB distributions, even though the distribution itself is not statistically different from the SNe Ic-BL sample. To really establish whether the hosts of these transients differ significantly or not, the number of studied hosts will have to be increased.

In all but two cases, the apparent galaxy size in the sky is much bigger than the slit width with which the spectrum was taken. The metallicities for these events can be considered as being measured at the explosion sites, though the spatial resolution naturally does not match the resolutions achieved when observing very close host galaxies with integral field units like VLT/MUSE (see e.g. Krühler et al. 2017; Izzo et al. 2017). Metallicities for the SN2006nx and SN2007I hosts are galaxy-averaged values as the galaxies are small in the sky. Detailed studies of nearby, well resolved host galaxies of GRB$\mathrm{SNe}$ with integral field spectroscopy have shown that the metallicities at the explosion sites do not differ appreciably from the host-averaged metallicities (e.g. Christensen et al. 2008; Levesque et al. 2011; Krühler et al. 2017; Izzo et al. 2017). The two galaxies are also physically quite small (and comparable to the sizes of GRB-SN hosts) and therefore we expect their average metallicities to be similar to the metallicity at the SN site.

We note that the metallicities measured and quoted in this paper represent oxygen abundances. On the other hand, the metallicities that are used in the computations of theoretical models are based on iron, which we cannot measure directly. In the following discussion we implicitly assume that iron scales with oxygen and the diagnostics that we use can be used as a proxy for the metal content in stars.

\section{Implications for progenitors}

Our findings potentially harbour important clues about the nature of the progenitors. In the context of the collapsar scenario (Woosley 1993; MacFadyen \& Woosley 1999; MacFadyen et al. 2001) the progenitor model needs to explain how to retain enough angular momentum in the core at the moment of explosion, while losing the $\mathrm{H}$ - and He-rich outermost layers (Woosley \& Bloom 2006; Modjaz et al. 2016). In a single star scenario, mass loss mechanisms typically also remove angular momentum, and are sensitive to the progenitor
Table 4. Metallicity measurements in the $\mathrm{O} 3 \mathrm{~N} 2$ and M08 calibrations.

\begin{tabular}{lccr}
\hline \hline & \multicolumn{2}{c}{$12+\log \left(\frac{\mathrm{O}}{\mathrm{H}}\right)$} & \\
\cline { 2 - 3 } SN/GRB & O3N2 & M08 & Ref \\
\cline { 2 - 3 } SN2005kr* & $8.24 \pm 0.01$ & $8.63 \pm 0.03$ & 1 \\
SN2005ks & $8.63 \pm 0.01$ & $8.87 \pm 0.03$ & 1 \\
SN2005nb & $8.44 \pm 0.03$ & $8.70 \pm 0.06$ & 2 \\
SN2006nx & $8.28 \pm 0.04$ & $8.48 \pm 0.06$ & 2 \\
SN2006qk* & $8.75 \pm 0.02$ & $8.82 \pm 0.05$ & 1 \\
SN2007I & $8.58 \pm 0.07$ & $8.80 \pm 0.07$ & 2 \\
SN2007ce & $7.99 \pm 0.02$ & $8.00 \pm 0.02$ & 2 \\
SN2007eb* & $8.26 \pm 0.07$ & $8.43 \pm 0.10$ & 1 \\
SN2007gx* & $8.85 \pm 0.06$ & $9.14 \pm 0.02$ & 1 \\
SN2007qw* & $8.19 \pm 0.01$ & $8.50 \pm 0.07$ & 1 \\
SN2008iu* & $8.05 \pm 0.03$ & $8.06 \pm 0.04$ & 1 \\
SN2010ah & $8.32 \pm 0.05$ & $8.55 \pm 0.10$ & 2 \\
SN2010ay & $8.16 \pm 0.02$ & $8.28 \pm 0.02$ & 2 \\
PTF10vgv & $8.67 \pm 0.06$ & $8.86 \pm 0.06$ & 2 \\
\hline GRB 980425 & $8.31 \pm 0.01$ & $8.50 \pm 0.02$ & 3 \\
GRB 030329 & $8.19 \pm 0.10$ & $8.28 \pm 0.04$ & 4 \\
GRB 031203A & $8.12 \pm 0.06$ & $8.08 \pm 0.10$ & 5 \\
GRB 060218 & $8.13 \pm 0.03$ & $8.28 \pm 0.04$ & 6 \\
GRB 100316D & $8.16 \pm 0.01$ & $8.30 \pm 0.02$ & 7 \\
GRB 161219B & $8.37 \pm 0.08$ & $8.56 \pm 0.04$ & 8 \\
\hline
\end{tabular}

Notes. SNe Ic-BL tagged with * are not a part of our original sample: they are part of the untargeted survey presented by Modjaz et al. (2011), but their classification is not confirmed. References point to the source material from which we collected the measurements of emission-line fluxes.

References. (1) Modjaz et al. (2011), (2) Sanders et al. (2012), (3) Krühler et al. (2017), (4) Gorosabel et al. (2005), (5) Guseva et al. (2011), (6) Wiersema et al. (2007), (7) Izzo et al. (2017) and (8) Cano et al. (2017a).

metallicity (stellar wind mass-loss rates approximately scale as $\approx Z^{0.8}$; Vink et al. 2001; Mokiem et al. 2007). Conversely, binaries provide a reservoir of angular momentum stored in the orbit, which can be tapped into through tidal interactions (Izzard et al. 2004; Detmers et al. 2008; de Mink et al. 2009), mass accretion (Cantiello et al. 2007; Eldridge et al. 2011; de Mink et al. 2013), and/or mergers (Tout et al. 2011; Zapartas et al. 2017). Moreover, binaries can increase the delay time between the star formation phase and the death of massive stars (Zapartas et al. 2017). Therefore certain binary products have on average more time to travel longer distances from their birth location, possibly resulting in larger offsets. On the other hand, if GRBs occur via a binary channel, then their preference for dense environments might actually lead to shorter merger time scales (e.g. van den Heuvel \& Portegies Zwart 2013).

Modjaz et al. (2016) have shown that there are significant spectral differences between SNe Ic-BL with and without GRBs, which suggests two different progenitor classes, or, possibly, a continuum of progenitors resulting at the extreme in the two different phenomena. Our findings, albeit based on a limited sample of hosts, seem to corroborate this idea.

A general study of the offsets of long GRB explosions was first performed by Bloom et al. (2002), who found that GRBs indeed occur close to the brightest, star-forming regions of their hosts (see also Sect. 3.2). A more detailed pixel-by-pixel analysis of resolved host images has confirmed the trend and in addition revealed that GRBs are found in brighter parts of their host 


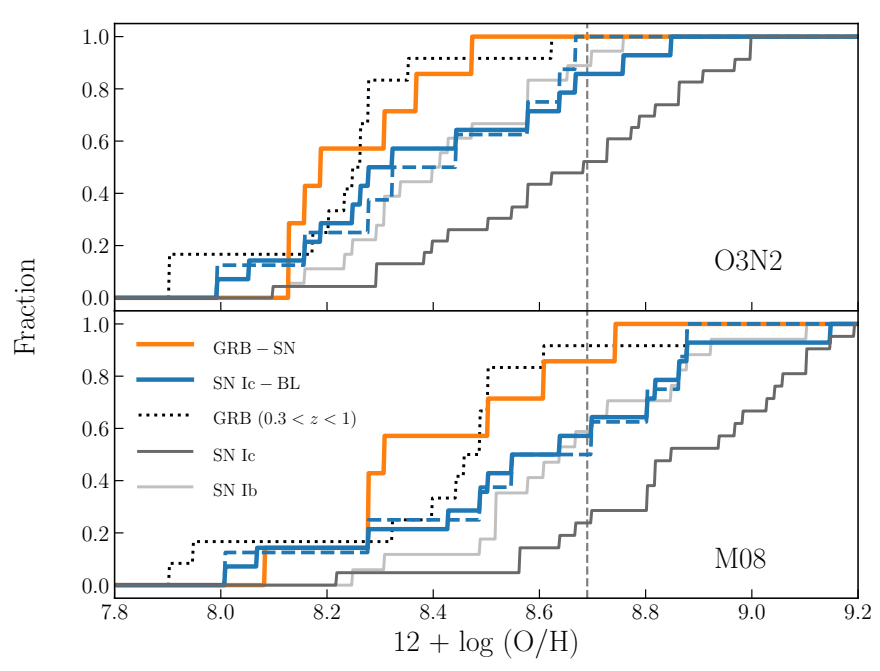

Fig. 5. Cumulative metallicity distributions of GRB-SN and SN Ic-BL samples: SNe Ic-BL without GRBs on average prefer environments with higher metallicities (see discussion in Sect. 4). For SNe Ic-BL, we plot the distribution including all hosts with metallicities in Table 4 (full line) and the distribution including only the hosts studied in this paper (dashed). Upper and lower plots: metallicities measured using the calibrations of (Pettini \& Pagel 2004; O3N2) and (Maiolino et al. 2008; M08), respectively. For comparison, we also plot the distribution of the $z<1$ sample of long GRB host galaxies (Krühler et al. 2015; Japelj et al. 2016a) and the samples of SN Ib and Ic host galaxies from Sanders et al. (2012) and Modjaz et al. (2011). The vertical dashed lines indicate solar oxygen abundance of $12+\log (\mathrm{O} / \mathrm{H})=8.69$ (Asplund et al. 2009).

galaxies with respect to a general class of core-collapse $\mathrm{SNe}$ (Fruchter et al. 2006; Kelly et al. 2008; Svensson et al. 2010; Lyman et al. 2017). The regions of the most recent star formation are expected to be brighter than the regions where more time has passed since the star formation episode, because the massive stars have not yet exploded. This result has therefore been interpreted as evidence for massive stellar progenitors and short progenitor lifetimes of long GRBs. The difference in the distribution of offsets of the SNe Ic-BL with and without GRBs that we find (Fig. 4) implies that the progenitors of the GRB-SNe may be shorter-lived stars with respect to SNe Ic-BL. The shorter lifetime could be the natural consequence of a more massive progenitor, either in the case of a single star evolution scenario or possibly resulting from stellar mergers. If the difference in the offsets indeed translates into more massive progenitors for GRB$\mathrm{SNe}$, this would be evidence against the scenario where the $\mathrm{SNe}$ Ic-BL without GRBs are the result of choking (damping) of the jet. In fact, we expect the choking of the jet to become progressively more efficient as the mass of the layers the jet has to plow through increases (e.g. Margutti et al. 2014, 2015; Modjaz et al. 2016).

Above, we implicitly assumed that the peak of the surface brightness traces young stellar populations. At low redshifts in particular, this may not necessarily be true; for example, in cases where the brightness is dominated by a bulge instead of a disk, the high surface-brightness regions are dominated by older stars. A measurement of the concentration of light $C \equiv 5 \log \left(r_{80} / r_{20}\right)$ (Kent 1985; Conselice et al. 2005) for our galaxies gives values of $C \lesssim 3$, suggesting that the light is indeed dominated by the contribution from disks and not from the bulge. Nevertheless, the fact remains that many galaxies in our sample are not really resolved and therefore we cannot measure the distances to the individual star-forming regions (as for example in the case of GRB 980425). It is therefore more accurate to interpret the offsets as the distances to the regions with the highest stellar densities and it follows from our analysis that GRB-SNe tend to occur closer to the densest regions in their host galaxies.

Both long GRBs and GRB-SNe have a systematically lower host metallicity than SNe Ic-BL (see also Graham \& Fruchter 2013). The lower host metallicity of GRBs fits naturally with the expectation of reduced wind mass and angular momentum losses with lower metallicity (Vink et al. 2001). Strong stellar winds could potentially spin down the progenitor whether its high rotation is primordial or due to binary interactions. The difference in the host metallicities might be an indication of the existence of two different channels for the formation of GRB-SNe and $\mathrm{SNe}$ Ic-BL or evidence that a GRB jet cannot be successfully produced in the case of high metallicity.

Recently, Barnes et al. (2018) performed a simulation of a jet-driven core-collapse event and demonstrated that a GRB engine can produce a long GRB and at the same time drive a $\mathrm{SN}$ explosion. The produced $\mathrm{SN}$ has characteristics typical of SN Ic-BL and its properties depend on the viewing angle. The study does not fully explore the parameter space and therefore it remains unclear whether the observed difference in the spectra of SNe Ic-BL and GRB-SNe (Modjaz et al. 2016) can be attributed solely to the viewing angle. The trends in the environment that we find suggest that the difference is not solely due to the viewing angle. It would be interesting to see how the progenitor mass and/or metallicity affect the successful break-out of the jet from the progenitor.

The metallicity distribution in Fig. 5 shows that SNe Ic-BL have environments more similar to SNe Ib than SNe Ic. One possible explanation could be that there are different mechanisms to remove the He lines from the spectra of SNe Ic and SNe Ic-BL. However, Modjaz et al. (2016) have shown that by artificially broadening the spectra of a normal SNe Ic, one finds good agreement with the spectrum of a SNe Ic-BL, which is surprising if the $\mathrm{He}$ is removed by a different mechanism. Another possibility is that the progenitors of SNe Ic-BL are more massive than those of normal SNe Ic, and they can therefore lose their He-rich layers to winds at lower metallicity, owing to their higher luminosity that drives the wind.

Finally, while it is indisputable that metallicity is an important factor in the GRB production efficiency, it is unclear what is the effect of other properties (e.g. Perley et al. 2016a). For example, Kelly et al. (2014) found that GRB hosts are smaller (and more star-forming) than the hosts of SNe Ib/c and SNe II of the same stellar mass (see also Japelj et al. 2016a). This implies that GRBs prefer dense stellar environments with a particularly intense star formation, where a higher fraction of massive stars, close binaries, or interactions between stars can be produced. These results are still tentative and should be explored in more detail. We do not have sufficient photometric and spectroscopic data at this point to do a similar analysis for our samples of GRBSN and SN Ic-BL hosts.

In the above discussion we have implicitly assumed that GRB-SNe are a subset of the long GRB class. There are three known nearby long GRBs for which an associated SN has not been found even after deep follow-up observational campaigns. These are GRB 060505, GRB 060614 (Fynbo et al. 2006; Gal-Yam et al. 2006; Della Valle et al. 2006; Xu et al. 2009) and GRB 111005A (Tanga et al. 2018). The long duration of GRBs 060505 and 060614 has been put into question (e.g. Ofek et al. 2007; Yang et al. 2015). On the other hand, the long nature of GRB 111005A seems to be better established. 
Table 5. Results of the two-sided AD test between metallicity distributions of different $\mathrm{SN}$ and long GRB samples.

\begin{tabular}{lcc}
\hline \hline & \multicolumn{2}{c}{ Chance probability $p_{\text {ch }}$} \\
SN/GRB & O3N2 & M08 \\
\hline GRB-SN - Ic-BL & 0.43 & 0.23 \\
GRB-SN - long GRB & 0.53 & 0.67 \\
GRB-SN - Ic & 0.001 & 0.0006 \\
GRB-SN - Ib & 0.07 & 0.02 \\
Long GRB - Ic-BL & 0.16 & 0.12 \\
Long GRB - Ic & 0.00001 & 0.00001 \\
Long GRB - Ib & 0.01 & 0.004 \\
Ic - Ic-BL & 0.004 & 0.004 \\
Ic - Ib & 0.004 & 0.02 \\
Ib - Ic-BL & 0.27 & 0.27 \\
\hline
\end{tabular}

Interestingly, this GRB has been found in a metal-rich environment with low ongoing star-formation rate (Tanga et al. 2018). If this class of SN-less GRBs is indeed real, then a different evolutionary channel is required to explain it.

\section{Conclusions}

It is not yet clear whether SNe Ic-BL with and without an accompanying GRB represent the same events seen at different inclination angles or if they are intrinsically different events resulting from different evolutionary channels. In order to shed light on this issue, we have done a systematic analysis of the host galaxies of SNe Ic-BL with and without an associated GRB explosion. The aim of this study is to find out whether there are any systematic differences between the environments in which these two types of explosions occur. A difference in the environment would indicate that not every SN Ic-BL is accompanied by a GRB and help us to better constrain the evolutionary channels that lead to each explosion. We collected a sample of 28 untargeted SNe Ic-BL and a sample of 8 GRB-SNe at $z<0.2$ and analysed the images of their host galaxies. We provide measurements of galaxy luminosities, galaxy sizes $\left(r_{50}\right.$ and $\left.r_{90}\right)$, and projected offsets between the explosions and the galaxy centres.

We find that the SN Ic-BL and GRB-SN hosts have similar luminosities and sizes. On the other hand, the projected offsets normalized by galaxy sizes of GRB-SNe seem to be skewed towards lower values with respect to the SNe Ic-BL. The difference is of low statistical significance (chance probability of $p_{\text {ch }}=0.07$ that the two distributions are drawn from the same parent distribution). Nevertheless, the result could indicate that GRB-SNe occur closer to the regions of the highest stellar densities in their host galaxies with respect to SNe Ic-BL.

We look for additional potential differences in the environments of the two classes, in particular we study the metallicity. Collecting the available spectral information of the host galaxies (i.e. values of emission-line fluxes), we estimated the gas-phase metallicities of the host galaxies of the two samples. Indeed there is a larger fraction of SN Ic-BL hosts with super-solar metallicities with respect to the host galaxies of GRB-SNe. The differences that we find suggest a genuine difference to exist between the progenitors of SNe Ic-BL and GRB-SNe and it is unlikely that the differences found in the spectral properties are only due to the effect of a viewing angle. It however remains to be understood whether the two types of explosions are produced through a similar or different evolutionary channel.
The progenitor star models for these explosions are still very uncertain and poorly constrained. Every observational constraint is helpful in testing, tightening, and improving them. The results presented in this paper show the potential of environment studies to provide such constraints. The sample of nearby, spectroscopically confirmed GRB-SNe is small and cannot be significantly increased. On the other hand, the sample of SNe Ic-BL is quite large but only $\sim 35 \%$ of their host galaxies have been observed spectroscopically. By increasing the number of available spectra and therefore the number of hosts with measured metallicities (and other properties like star formation rates, ages of stellar populations, etc.), the properties of these two classes of explosions will be much better constrained.

Acknowledgements. We are grateful to Enrico Ramirez-Ruiz for fruitful discussion. JJ and LK acknowledge support from NOVA and NWO-FAPESP grant for advanced instrumentation in astronomy. SDV acknowledges the support of the French National Research Agency (ANR) under contract ANR-16-CE310003 BEaPro (P.I.: SDV). SdM has received funding under the European Unions Horizon 2020 research and innovation programme from the European Research Council (ERC; Grant agreement No. 715063). Funding for SDSS-III has been provided by the Alfred P. Sloan Foundation, the Participating Institutions, the National Science Foundation, and the U.S. Department of Energy Office of Science. The SDSS-III web site is http://www . sdss3. org/. SDSS-III is managed by the Astrophysical Research Consortium for the Participating Institutions of the SDSS-III Collaboration including the University of Arizona, the Brazilian Participation Group, Brookhaven National Laboratory, Carnegie Mellon University, University of Florida, the French Participation Group, the German Participation Group, Harvard University, the Instituto de Astrofisica de Canarias, the Michigan State/Notre Dame/JINA Participation Group, Johns Hopkins University, Lawrence Berkeley National Laboratory, Max Planck Institute for Astrophysics, Max Planck Institute for Extraterrestrial Physics, New Mexico State University, New York University, Ohio State University, Pennsylvania State University, University of Portsmouth, Princeton University, the Spanish Participation Group, University of Tokyo, University of Utah, Vanderbilt University, University of Virginia, University of Washington, and Yale University. The PanSTARRS1 Surveys (PS1) and the PS1 public science archive have been made possible through contributions by the Institute for Astronomy, the University of Hawaii, the Pan-STARRS Project Office, the Max-Planck Society and its participating institutes, the Max Planck Institute for Astronomy, Heidelberg and the Max Planck Institute for Extraterrestrial Physics, Garching, The Johns Hopkins University, Durham University, the University of Edinburgh, the Queen's University Belfast, the Harvard-Smithsonian Center for Astrophysics, the Las Cumbres Observatory Global Telescope Network Incorporated, the National Central University of Taiwan, the Space Telescope Science Institute, the National Aeronautics and Space Administration under Grant No. NNX08AR22G issued through the Planetary Science Division of the NASA Science Mission Directorate, the National Science Foundation Grant No. AST-1238877, the University of Maryland, Eotvos Lorand University (ELTE), the Los Alamos National Laboratory, and the Gordon and Betty Moore Foundation. We acknowledge ESA Gaia, DPAC and the Photometric Science Alerts Team (http://gsaweb.ast.cam . ac.uk/alerts). This research made use of Astropy, a community-developed core Python package for Astronomy (Astropy Collaboration 2013).

\section{References}

Alam, S., Albareti, F. D., Allende Prieto, C., et al. 2015, ApJS, 219, 12

Alexander, K. D., Laskar, T., \& Berger, E. 2016, GRB Coordinates Network. Circular Service, No., 20313, \#1

Anderson, J. P., Covarrubias, R. A., James, P. A., Hamuy, M., \& Habergham, S. M. 2010, MNRAS, 407, 2660

Arcavi, I., Horesh, A., \& Sesar, B. 2010, ATel, 3095

Asplund, M., Grevesse, N., Sauval, A. J., \& Scott, P. 2009, ARA\&A, 47, 481

Astropy Collaboration (Robitaille, T. P., et al.) 2013, A\&A, 558, A33

Barnes, J., Duffell, P. C., Liu, Y., et al. 2018, ApJ, 860, 38

Bassett, B., Becker, A., Brewington, H., et al. 2006, Cent. Bureau Electron. Tel., 1,743

Ben-Ami, S., Gal-Yam, A., Filippenko, A. V., et al. 2012, ApJ, 760, L33

Bertin, E. 2011, in Astronomical Data Analysis Software and Systems XX, eds.

I. N. Evans, A. Accomazzi, D. J. Mink, \& A. H. Rots, ASP Conf. Ser., 442, 435

Bertin, E., \& Arnouts, S. 1996, A\&AS, 117, 393

Blanchard, P. K., Berger, E., \& Fong, W.-F. 2016, ApJ, 817, 144

Bloom, J. S., Kulkarni, S. R., \& Djorgovski, S. G. 2002, AJ, 123, 111 
Cano, Z. 2013, MNRAS, 434, 1098

Cano, Z., Bersier, D., Guidorzi, C., et al. 2011, ApJ, 740, 41

Cano, Z., Izzo, L., de Ugarte Postigo, A., et al. 2017a, A\&A, 605, A107

Cano, Z., Wang, S.-Q., Dai, Z.-G., \& Wu, X.-F. 2017b, Adv. Astron., 2017, 8929054

Cantiello, M., Yoon, S.-C., Langer, N., \& Livio, M. 2007, A\&A, 465, L29

Cao, Y., Kasliwal, M. M., Arcavi, I., et al. 2013, ApJ, 775, L7

Chambers, K. C., Magnier, E. A., Metcalfe, N., et al. 2016, ArXiv e-prints [arXiv: 1612.05560]

Christensen, L., Vreeswijk, P. M., Sollerman, J., et al. 2008, A\&A, 490, 45

Conselice, C. J., Vreeswijk, P. M., Fruchter, A. S., et al. 2005, ApJ, 633, 29

Corsi, A., Ofek, E. O., Gal-Yam, A., et al. 2012, ApJ, 747, L5

Corsi, A., Gal-Yam, A., Kulkarni, S. R., et al. 2016, ApJ, 830, 42

de Mink, S. E., Cantiello, M., Langer, N., et al. 2009, A\&A, 497, 243

de Mink, S. E., Langer, N., Izzard, R. G., Sana, H., \& de Koter, A. 2013, ApJ, 764, 166

Della Valle, M., Chincarini, G., Panagia, N., et al. 2006, Nature, 444, 1050

Detmers, R. G., Langer, N., Podsiadlowski, P., \& Izzard, R. G. 2008, A\&A, 484, 831

Drake, A. J., Djorgovski, S. G., Mahabal, A., et al. 2010, Cent. Bureau Electron. Tel., 1, 2224

Eldridge, J. J., Langer, N., \& Tout, C. A. 2011, MNRAS, 414, 3501

Filippenko, A. V. 1997, ARA\&A, 35, 309

Fruchter, A. S., Levan, A. J., Strolger, L., et al. 2006, Nature, 441, 463

Fynbo, J. P. U., Watson, D., Thöne, C. C., et al. 2006, Nature, 444, 1047

Gal-Yam, A., Fox, D. B., Price, P. A., et al. 2006, Nature, 444, 1053

Gal-Yam, A., Arcavi, I., Xu, D., et al. 2010, ATel, 2817

Galama, T. J., Vreeswijk, P. M., van Paradijs, J., et al. 1998, Nature, 395, 670

Ghirlanda, G., Ghisellini, G., Salvaterra, R., et al. 2013, MNRAS, 428, 1410

Gorosabel, J., Pérez-Ramírez, D., Sollerman, J., et al. 2005, A\&A, 444, 711

Graham, J. F., \& Fruchter, A. S. 2013, ApJ, 774, 119

Granot, J. 2007, Rev. Mex. Astron. Astrofis. 27, 140

Graur, O., Bianco, F. B., Modjaz, M., et al. 2017, ApJ, 837, 121

Groh, J. H., Georgy, C., \& Ekström, S. 2013, A\&A, 558, L1

Guetta, D., \& Della Valle, M. 2007, ApJ, 657, L73

Guillochon, J., Parrent, J., Kelley, L. Z., \& Margutti, R. 2017, ApJ, 835, 64

Guseva, N. G., Izotov, Y. I., Fricke, K. J., \& Henkel, C. 2011, A\&A, 534, A84

Hammer, F., Flores, H., Schaerer, D., et al. 2006, A\&A, 454, 103

Heger, A., Fryer, C. L., Woosley, S. E., Langer, N., \& Hartmann, D. H. 2003, ApJ, 591, 288

Hjorth, J., Sollerman, J., Møller, P., et al. 2003, Nature, 423, 847

Holmberg, E. 1958, Meddelanden fran Lunds Astronomiska Observatorium Series II, 136, 1

Izzard, R. G., Ramirez-Ruiz, E., \& Tout, C. A. 2004, MNRAS, 348, 1215

Izzo, L., Thöne, C. C., Schulze, S., et al. 2017, MNRAS, 472, 4480

Japelj, J., Vergani, S. D., Salvaterra, R., et al. 2016a, A\&A, 590, A129

Japelj, J., Vergani, S. D., Salvaterra, R., Hunt, L. K., \& Mannucci, F. 2016b, A\&A, 593, A115

Jin, C. C., Cao, Y., Bian, F.-Y., et al. 2007, IAU Circ., 1, 8798

Kann, D. A., Schady, P., Olivares, E. F., et al. 2016, A\&A, submitted, [arxiv 1606.06791]

Kelly, P. L., \& Kirshner, R. P. 2012, ApJ, 759, 107

Kelly, P. L., Kirshner, R. P., \& Pahre, M. 2008, ApJ, 687, 1201

Kelly, P. L., Filippenko, A. V., Modjaz, M., \& Kocevski, D. 2014, ApJ, 789, 23

Kent, S. M. 1985, ApJS, 59, 115

Kewley, L. J., \& Dopita, M. A. 2002, ApJS, 142, 35

Kewley, L. J., \& Ellison, S. L. 2008, ApJ, 681, 1183

Kron, R. G. 1980, ApJS, 43, 305

Krühler, T., Malesani, D., Fynbo, J. P. U., et al. 2015, A\&A, 581, A125

Krühler, T., Kuncarayakti, H., Schady, P., et al. 2017, A\&A, 602, A85

Kumar, P., \& Zhang, B. 2015, Phys. Rep., 561, 1

Langer, N. 2012, ARA\&A, 50, 107

Laskar, T., Coppejans, D. L., Margutti, R., \& Alexander, K. D. 2017, GRB Coordinates Network. Circular Service, No 2216, \#1

Law, N. M., Kulkarni, S. R., Dekany, R. G., et al. 2009, PASP, 121, 1395

Lazzati, D., Morsony, B. J., Blackwell, C. H., \& Begelman, M. C. 2012, ApJ, 750,68

Levesque, E. M., Berger, E., Soderberg, A. M., \& Chornock, R. 2011, ApJ, 739, 23

Lyman, J. D., Bersier, D., James, P. A., et al. 2016, MNRAS, 457, 328

Lyman, J. D., Levan, A. J., Tanvir, N. R., et al. 2017, MNRAS, 467, 1795

MacFadyen, A. I., \& Woosley, S. E. 1999, ApJ, 524, 262
MacFadyen, A. I., Woosley, S. E., \& Heger, A. 2001, ApJ, 550, 410

Maeder, A., \& Meynet, G. 2000, ARA\&A, 38, 143

Maiolino, R., Nagao, T., Grazian, A., et al. 2008, A\&A, 488, 463

Malesani, D., Tagliaferri, G., Chincarini, G., et al. 2004, ApJ, 609, L5

Margutti, R., Milisavljevic, D., Soderberg, A. M., et al. 2014, ApJ, 797, 107

Margutti, R., Guidorzi, C., Lazzati, D., et al. 2015, ApJ, 805, 159

Mazzali, P. A., Deng, J., Pian, E., et al. 2006, ApJ, 645, 1323

Modjaz, M., Kewley, L., Kirshner, R. P., et al. 2008, AJ, 135, 1136

Modjaz, M., Kewley, L., Bloom, J. S., et al. 2011, ApJ, 731, L4

Modjaz, M., Blondin, S., Kirshner, R. P., et al. 2014, AJ, 147, 99

Modjaz, M., Liu, Y. Q., Bianco, F. B., \& Graur, O. 2016, ApJ, 832, 108

Mokiem, M. R., de Koter, A., Vink, J. S., et al. 2007, A\&A, 473, 603

Moretti, S., Tomaselli, S., Puckett, T., et al. 2007, IAU Circ., 1, 8834

Ofek, E. O., Cenko, S. B., Gal-Yam, A., et al. 2007, ApJ, 1, 662, 1129

Ofek, E. O., Howell, D. A., Kasliwal, M. M., et al. 2010, Cent. Bureau Electron. Tel., 1, 2198

Peng, C. Y., Ho, L. C., Impey, C. D., \& Rix, H.-W. 2010, AJ, 139, 2097

Perley, D. A., Niino, Y., Tanvir, N. R., Vergani, S. D., \& Fynbo, J. P. U. 2016a, Space Sci. Rev., 202, 111

Perley, D. A., Tanvir, N. R., Hjorth, J., et al. 2016b, ApJ, 817, 8

Pettini, M., \& Pagel, B. E. J. 2004, MNRAS, 348, L59

Pian, E., Tomasella, L., Cappellaro, E., et al. 2017, MNRAS, 466, 1848

Pier, J. R., Munn, J. A., Hindsley, R. B., et al. 2003, AJ, 125, 1559

Planck Collaboration XVI. 2014, A\&A, 571, A16

Polshaw, J., Benitez, S., Taubenberger, S., et al. 2014, ATel, 6094

Prentice, S. J., Mazzali, P. A., Pian, E., et al. 2016, MNRAS, 458, 2973

Quimby, R., Mondol, P., Castro, F., Roman, B., \& Rostopchin, S. 2006, IAU Circ., 1, 8657

Quimby, R., Odewahn, S. C., Terrazas, E., Rau, A., \& Ofek, E. O. 2007, Cent. Bureau Electron. Tel., 1, 953

Sako, M., Bassett, B., Becker, A. C., et al. 2018, PASP, 130, 988

Salvaterra, R., Campana, S., Vergani, S. D., et al. 2012, ApJ, 749, 68

Sanders, N. E., Soderberg, A. M., Levesque, E. M., et al. 2012, ApJ, 758, 132

Schlafly, E. F., \& Finkbeiner, D. P. 2011, ApJ, 737, 103

Shibuya, T., Ouchi, M., \& Harikane, Y. 2015, ApJS, 219, 15

Singer, L. P., Cenko, S. B., Kasliwal, M. M., et al. 2013, ApJ, 776, L34

Singer, L. P., Kasliwal, M. M., Ferretti, R., et al. 2015, GRB Coordinates Network. Circular Service. No. 18497, 18566

Smith, N., Li, W., Filippenko, A. V., \& Chornock, R. 2011, MNRAS, 412, 1522

Soderberg, A. 2007, PhD Thesis, California Institute of Technology, USA

Soderberg, A. M., Kulkarni, S. R., Berger, E., et al. 2004, Nature, 430, 648

Soderberg, A. M., Kulkarni, S. R., Nakar, E., et al. 2006, Nature, 442, 1014

Soderberg, A. M., Chakraborti, S., Pignata, G., et al. 2010, Nature, 463, 513

Sollerman, J., Östlin, G., Fynbo, J. P. U., et al. 2005, New A, 11, 103

Starling, R. L. C., Wiersema, K., Levan, A. J., et al. 2011, MNRAS, 411, 2792

Svensson, K. M., Levan, A. J., Tanvir, N. R., Fruchter, A. S., \& Strolger, L.-G. 2010, MNRAS, 405, 57

Tanga, M., Krühler, T., Schady, P., et al. 2018, A\&A, 615, A136

Taubenberger, S., Valenti, S., Benetti, S., et al. 2009, MNRAS, 397, 677

Taylor, G. B., Frail, D. A., Berger, E., \& Kulkarni, S. R. 2004, ApJ, 609, L1

Thöne, C. C., de Ugarte Postigo, A., García-Benito, R., et al. 2015, MNRAS, 451, L65

Tinney, C., Stathakis, R., Cannon, R., et al. 1998, IAU Circ., 1, 6896

Tout, C. A., Wickramasinghe, D. T., Lau, H. H.-B., Pringle, J. E., \& Ferrario, L. 2011, MNRAS, 410, 2458

Unterborn, C. T., \& Ryden, B. S. 2008, ApJ, 687, 976

van den Heuvel, E. P. J., \& Portegies Zwart, S. F. 2013, ApJ, 779, 114

Van Dyk, S. D., Zheng, W., Brink, T. G., et al. 2018, ApJ, 860, 90

van Eerten, H. 2018, Int. J. Mod. Phys. D, accepted [arXiv:1801.01848]

Vergani, S. D., Salvaterra, R., Japelj, J., et al. 2015, A\&A, 581, A102

Vergani, S. D., Palmerio, J., Salvaterra, R., et al. 2017, A\&A, 599, A120

Vink, J. S., de Koter, A., \& Lamers, H. J. G. L. M. 2001, A\&A, 369, 574

Wang, L., \& Wheeler, J. C. 2008, ARA\&A, 46, 433

Wiersema, K., Savaglio, S., Vreeswijk, P. M., et al. 2007, A\&A, 464, 529

Woosley, S. E. 1993, ApJ, 405, 273

Woosley, S. E., \& Bloom, J. S. 2006, ARA\&A, 44, 507

Woosley, S. E., Heger, A., \& Weaver, T. A. 2002, Rev. Mod. Phys., 74, 1015

Xu, D., Starling, R. L. C., Fynbo, J. P. U., et al. 2009, ApJ, 696, 971

Yang, B., Jin, Z.-P., Li, X., et al. 2015, Nat. Commun., 6, 7323

Yoon, S.-C., Langer, N., \& Norman, C. 2006, A\&A, 460, 199

Zapartas, E., de Mink, S. E., Izzard, R. G., et al. 2017, A\&A, 601, A29

Zheng, C., Romani, R. W., Sako, M., et al. 2008, AJ, 135, 1766 


\section{Appendix A: Deprojected offsets}

We calculate deprojected offsets of explosion sites from the galaxy centres by accounting for the position angle (PA) and the inclination angle under which we view the galaxies. We estimate inclinations by assuming that galaxies can be represented as oblate spheroids (Holmberg 1958):

$\cos i=\frac{(b / a)^{2}-q^{2}}{1-q^{2}}$,

where $i$ is an inclination angle $\left(i=0^{\circ}\right.$ and $90^{\circ}$ correspond to galaxies seen face-on and edge-on, respectively), $b / a$ is the measured axial ratio and $q$ the intrinsic axial ratio. The values of $\mathrm{PA}$ and $b / a$ are obtained from our Galfit modelling - in case the galaxy is modelled with two components with significantly different values, then PA and $b / a$ of the more extended component are assumed for the measurement of the deprojected offset. We assume a typical intrinsic axial ratio of $q=0.2$ (see e.g. Unterborn \& Ryden 2008). If the measured axial ratio $b / a<q$, then we discard the galaxy from further analysis. This condition depends on our choice for the value of $q$. We note that the uncertainty in the measurement of inclination using this method is especially high at high inclinations (i.e. low $b / a$ ): it is indeed better to discard these systems in further analyses as the introduced uncertainty can be very high. If a galaxy is of irregular morphology (or the $b / a$ and PA values are poorly constrained in the fit) we assume that the galaxy is spherical and we do not apply the deprojection.

Once the inclination and position angles are known, we apply the deprojection. We firstly rotate the coordinate system for the $\mathrm{PA}$, so that the long axis of the galaxy is parallel to the $y$-axis, then we apply the deprojection $(y / \cos i)$ and calculate the deprojected offset. The values are given in Table A.1.

In Fig. A.1 we compare the projected and deprojected offsets of our two samples. The trend of GRB-SNe to be found closer to their host galaxy centres persists when deprojected offsets are considered. The AD test gives the chance probability $p_{\mathrm{AD}}=0.25$ and 0.1 that the distributions of the deprojected offsets and the normalized deprojected offsets are the same, respectively. Given the large uncertainties in the measurements of inclinations, we do not perform a more detailed MC simulation to estimate the chance probabilities. We note that the assumption we make for the value of $q$ may not give very accurate values of inclination for individual galaxies but should work well for large samples. Given that our samples (especially the GRB-SN sample) are small and that we are analysing a selected sample of galaxies, this might introduce a systematic uncertainty into our measurements.
Table A.1. Geometric properties of the host galaxies: the axial ratio $b / a$, the position angle PA (measured with respect to the north in the counter clockwise direction) and the inclination $i$.

\begin{tabular}{|c|c|c|c|c|}
\hline SN/GRB & $b / a$ & $\begin{array}{c}\text { PA } \\
\text { (deg) }\end{array}$ & $\begin{array}{c}i \\
(\mathrm{deg})\end{array}$ & $\begin{array}{r}\text { offset }_{\text {depr }} \\
(\mathrm{kpc})\end{array}$ \\
\hline SN2005ks & $0.31 \pm 0.05$ & $-10 \pm 4$ & 76 & 2.12 \\
\hline SN2005nb & $0.41 \pm 0.02$ & $38 \pm 3$ & 69 & 6.14 \\
\hline SDSS-IISN14475 & $0.11 \pm 0.18$ & $-5 \pm 10$ & - & * \\
\hline SN2006nx & $0.74 \pm 0.04$ & $75 \pm 10$ & 43 & 5.69 \\
\hline SN2007bg & - & - & - & - \\
\hline \multirow[t]{2}{*}{$\mathrm{SN} 2007 \mathrm{ce}^{a}$} & $<1$ & $58 \pm 13$ & 0 & $0.65^{\dagger}$ \\
\hline & $0.72 \pm 0.04$ & $10 \pm 3$ & 45 & 4.00 \\
\hline SN2007I & $0.61 \pm 0.01$ & $-36 \pm 2$ & 54 & 1.68 \\
\hline PTF10aavz & $0.56 \pm 0.05$ & $76 \pm 2$ & 56 & 5.57 \\
\hline SN2010ah & $0.14 \pm 0.01$ & $7 \pm 1$ & - & $*$ \\
\hline SN2010ay & $0.61 \pm 0.06$ & $60 \pm 5$ & 54 & 0.24 \\
\hline PTF10qts & $0.79 \pm 0.15$ & $49 \pm 30$ & 40 & 2.07 \\
\hline PTF10vgv & $0.45 \pm 0.02$ & $45 \pm 2$ & 66 & 2.02 \\
\hline PTF10xem & $0.48 \pm 0.03$ & $-40 \pm 5$ & 64 & 6.51 \\
\hline PTF11 cmh & $0.73 \pm 0.05$ & $-52 \pm 10$ & 44 & 5.82 \\
\hline PTF11lbm & $0.20 \pm 0.02$ & $-71 \pm 1$ & - & $*$ \\
\hline PTF12as & $0.79 \pm 0.03$ & $-78 \pm 30$ & 39 & 3.65 \\
\hline PTF13alq & $<0.15$ & $45 \pm 25$ & 0 & $0.90^{\dagger}$ \\
\hline PTF13ebw & $0.45 \pm 0.02$ & $-27 \pm 2$ & 66 & 10.92 \\
\hline PTF13u & $0.83 \pm 0.01$ & $37 \pm 7$ & 35 & 13.86 \\
\hline LSQ14bef & $0.24 \pm 0.01$ & $-78 \pm 2$ & 82 & 8.08 \\
\hline PTF14dby & $<1$ & $50 \pm 140$ & 0 & $0.75^{\dagger}$ \\
\hline PTF14gaq & $0.62 \pm 0.05$ & $-85 \pm 7$ & 53 & 5.75 \\
\hline \multirow[t]{2}{*}{ iPTF15dld $^{a}$} & $<1$ & $-85 \pm 151$ & 0 & $0.20^{\dagger}$ \\
\hline & $0.17 \pm 0.02$ & $-42 \pm 2$ & - & * \\
\hline SN2016coi & $0.76 \pm 0.02$ & $-42 \pm 4$ & 42 & 2.95 \\
\hline SN2016dst & - & - & 0 & $1.08^{\dagger}$ \\
\hline SN2017dcc & $0.58 \pm 0.01$ & $-78 \pm 3$ & 56 & 4.06 \\
\hline SN2017fgk & $0.30 \pm 0.01$ & $61 \pm 1$ & 77 & 0.82 \\
\hline GRB 980425 & $0.84 \pm 0.02$ & $-14 \pm 2$ & 34 & 2.33 \\
\hline GRB 030329 & $0.77 \pm 0.05$ & $-82 \pm 3$ & 39 & 0.93 \\
\hline GRB 031203A & $0.83 \pm 0.03$ & $87 \pm 5$ & 35 & 0.62 \\
\hline GRB 060218 & $<1$ & $46 \pm 30$ & 0 & $0.08^{\dagger}$ \\
\hline GRB 100316D & - & - & 0 & $2.48^{\dagger}$ \\
\hline GRB 130702A & $0.54 \pm 0.02$ & $20 \pm 3$ & 59 & 0.92 \\
\hline GRB 161219B & $0.11 \pm 0.05$ & $26 \pm 3$ & - & * \\
\hline GRB 171205A & $0.81 \pm 0.01$ & $-62 \pm 5$ & 43 & 7.82 \\
\hline
\end{tabular}

Notes. The calculated deprojected offset (with respect to the centre of the galaxy) is given in the final column. We do not provide errors for $i$ and offset $t_{\text {depr }}$ due to large uncertainties introduced in the measurement of the former. *Galaxy is not included in the deprojection study because $b / a<0.2$. ${ }^{\dagger}$ Galaxy assumed to be a spheroid observed at $i=0$, i.e. no deprojection is applied to the offset. ${ }^{(a)}$ The first line corresponds to the measurement assuming the host galaxy is only the bright (blue) region near the $\mathrm{SN}$ explosion. The second line is obtained in cases where the host galaxy is the whole complex. 
A\&A 617, A105 (2018)
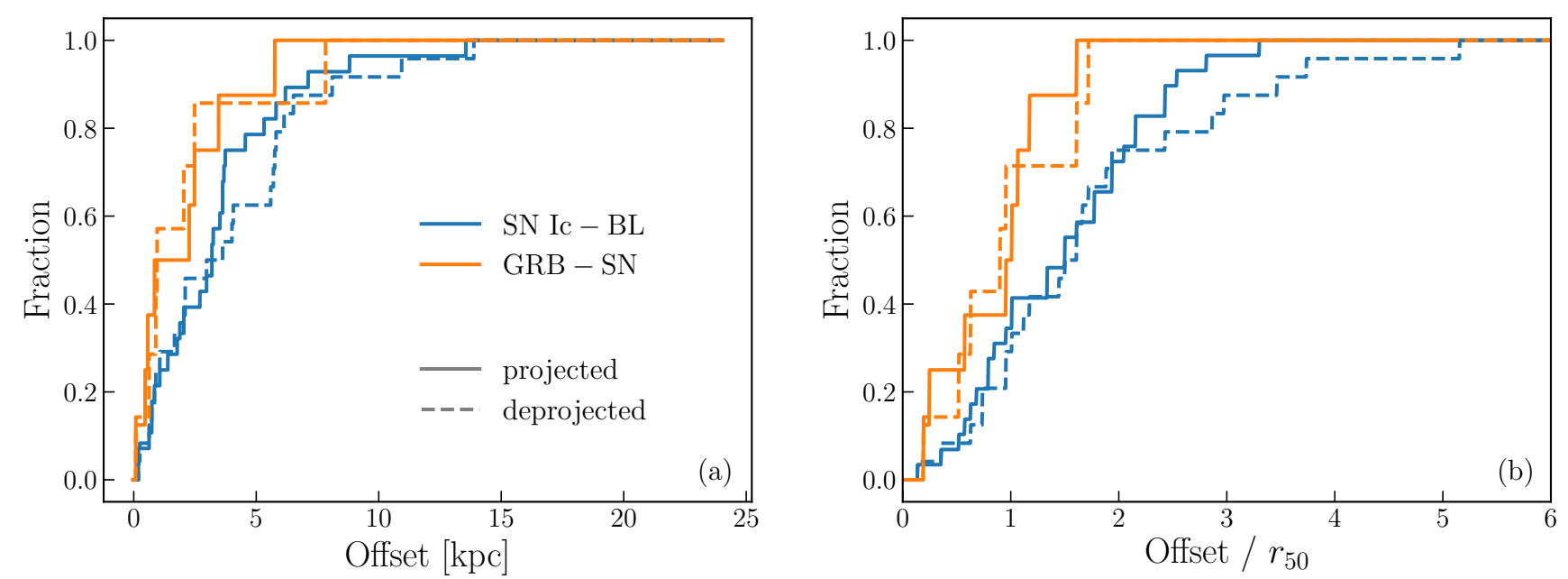

Fig. A.1. Comparing cumulative distributions of panel $a$ : offsets and panel $b$ : normalized offsets of the SN Ic-BL and GRB-SN samples. Dashed lines show distributions of deprojected offsets, where the geometric properties of the galaxies (see Table A.1) have been taken into account. 